\title{
Relative fundamental groups and rational points
}

\author{
Christopher Lazda $(*)$
}

ABSTRACT - In this paper we define a relative rigid fundamental group, which associates to a section of a smooth and proper morphism $f: X \rightarrow S$ in characteristic $p$, a Hopf algebra in the ind-category of overconvergent $F$-isocrystals on $S$. We prove a base change property, which says that the fibres of this object are the Hopf algebras of the rigid fundamental groups of the fibres of $f$. We explain how to use this theory to define period maps as Kim does for varieties over number fields, and show in certain cases that the targets of these maps can be interpreted as varieties.

Mathematics Subject Classification (2010). 11G35; 14G05, 14 F35.

KEYwoRDs. Unipotent fundamental group, non-abelian Jacobian.

\section{Contents}

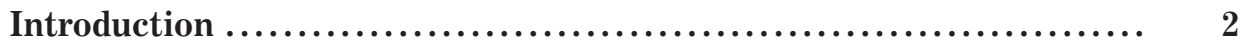

1. Relative de Rham fundamental groups..................... 5

1.1 - The relative fundamental group and its pro-nilpotent Lie algebra ... 6

1.2 - An algebraic proof of Theorem $1.6 \quad \ldots \ldots \ldots \ldots \ldots \ldots \ldots \ldots \ldots . . \ldots$

2. Path torsors, non-abelian crystals and period maps ........... 16

2.1 - Torsors in Tannakian categories $\ldots \ldots \ldots \ldots \ldots \ldots \ldots \ldots \ldots \ldots . \ldots \ldots$

2.2 - Path torsors under relative fundamental groups $\ldots \ldots \ldots \ldots \ldots \ldots .21$

3. Crystalline fundamental groups of smooth families in char $p \ldots \ldots . \quad 22$

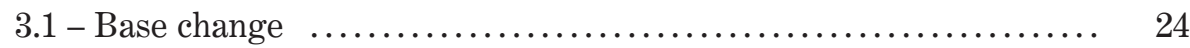

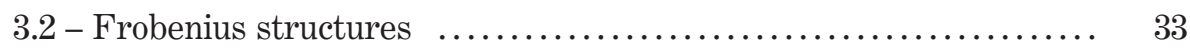

3.3 - Cohomology and period maps $\ldots \ldots \ldots \ldots \ldots \ldots \ldots \ldots \ldots \ldots \ldots \ldots \ldots \ldots \ldots \ldots \ldots$

(*) Indirizzo dell'A.: Department of Mathematics, Room 6.13 Huxley Building, 180 Queen's Gate, Imperial College, London, SW7 2AZ UK.

The author was supported by an EPSRC Doctoral Training Grant during the writing of this paper.

E-mail: c.lazda10@imperial.ac.uk 


\section{Introduction}

Let $K$ be a number field and let $C / K$ be a smooth, projective curve of genus $g>1$, with Jacobian $J$. Then a famous theorem of Faltings states that the set $C(K)$ of $K$-rational points on $C$ is finite. The group $J(K)$ is finitely generated, and under the assumption that its rank is strictly less than $g$, Chabauty in [14] was able to prove this theorem using elementary methods as follows. Let $v$ be a place of $K$, of good reduction for $C$, and denote by $C_{v}, J_{v}$ the base change to $K_{v}$. Then Chabauty defines a homomorphism

$$
\log : J\left(K_{v}\right) \rightarrow H^{0}\left(J_{v}, \Omega_{J_{v} / K_{v}}^{1}\right)
$$

and shows that there exists a non zero linear functional on $H^{0}\left(J_{v}, \Omega_{J_{v} / K_{v}}^{1}\right)$ which vanishes on the image of $J(K)$. He then proves that pulling this back to $J\left(K_{v}\right)$ gives an analytic function on $J\left(K_{v}\right)$, which is not identically zero on $C\left(K_{v}\right)$, and which vanishes on $J(K)$. Hence $C(K) \subset C\left(K_{v}\right) \cap J(K)$ must be finite as it is contained in the zero set of a non-zero analytic function on $C\left(K_{v}\right)$.

In [29], Kim describes what he calls a 'non-abelian lift' of this method. Fix a point $p \in C(K)$. By considering the Tannakian category of integrable connections on $C_{v}$, one can define a 'de Rham fundamental group' $U^{\mathrm{dR}}=\pi_{1}^{\mathrm{dR}}\left(C_{v}, p\right)$, which is a pro-unipotent group scheme over $K_{v}$, as well as, for any other $x \in C\left(K_{v}\right)$, path torsors $P^{\mathrm{dR}}(x)=\pi_{1}^{\mathrm{dR}}\left(C_{v}, x, p\right)$ which are right torsors under $U^{\mathrm{dR}}$. These group schemes and torsors come with extra structure, namely that of a Hodge filtration and, by comparison with the crystalline fundamental group of the reduction of $C_{v}$, a Frobenius action. He then shows that such torsors are classified by $U^{\mathrm{dR}} / F^{0}$, and hence one can define 'period maps'

$$
j_{n}: C\left(K_{v}\right) \rightarrow U_{n}^{\mathrm{dR}} / F^{0}
$$

where $U_{n}^{\mathrm{dR}}$ is the $n$th level nilpotent quotient of $U^{\mathrm{dR}}$. If $n=2$ then $j_{n}$ is just the composition of the above log map with the inclusion $C\left(K_{v}\right) \rightarrow J\left(K_{v}\right)$. By analysing the image of this map, he is able to prove finiteness of $C(K)$ under certain conditions, namely if the dimension of $U_{n}^{\mathrm{dR}} / F^{0}$ is greater than the dimension of the target of a global period map defined using the category of lisse étale sheaves on $C$. Moreover, when $n=2$, this condition on dimensions is essentially Chabauty's condition that $\operatorname{rank}_{\mathrm{Z}} J(K)<$ genus $(C)$ (modulo the Tate-Shafarevich conjecture).

Our interest lies in trying to develop a function field analogue of these ideas. The analogy between function fields in one variable over finite fields and number fields has been a fruitful one throughout modern number theory, and indeed the analogue of Mordell's conjecture was first proven for function fields by Grauert. In this paper we discuss the problem of defining a good analogue of the global period map. This is defined in [29] using the Tannakian category of lisse $Q_{p}$ sheaves on $X$, and this approach will not work in the function field setting. Neither $p$-adic nor $\ell$ adic étale cohomology will give satisfactory answers, the first because, for example, 
the resulting fundamental group will be moduli dependent, i.e. will not be locally constant in families (see for example [34]), and the second because the $\ell$-adic topology on the resulting target spaces for period maps will not be compatible with the $p$-adic topology on the source varieties. Instead we will work with the category of overconvergent $F$-isocrystals.

Let $K$ be a finite extension of $\mathbb{F}_{p}(t)$, and let $k$ be the field of constants of $K$, i.e. the algebraic closure of $F_{p}$ inside $K$. Let $\bar{S}$ be the unique smooth projective, geometrically irreducible curve over $k$ whose function field is $K$. If $C / K$ is a smooth, projective, geometrically integral curve then one can choose a regular model for $C$. This is a regular, proper surface $\bar{X} / k$, equipped with a flat, proper morphism $f: \bar{X} \rightarrow \bar{S}$ whose generic fibre is $C / K$. Let $S \subset \bar{S}$ be the smooth locus of $f$, and denote by $f$ also the pullback $f: X \rightarrow S$. The idea is to construct, for any section $p$ of $f$, a 'non-abelian isocrystal' on $S$ whose fibre at any closed point $s$ 'is' the rigid fundamental group $\pi_{1}^{\text {rig }}\left(X_{s}, p_{s}\right)$. The idea behind how to construct such an object is very simple.

Suppose that $f: X \rightarrow S$ is a Serre fibration of topological spaces, with connected base and fibres. If $p$ is a section, then for any $s \in S$ the homomorphism $\pi_{1}(X, p(s)) \rightarrow \pi_{1}(S, s)$ is surjective, and $\pi_{1}(S, s)$ acts on the kernel via conjugation. This corresponds to a locally constant sheaf of groups on $S$, and the fibre over any point $s \in S$ is just the fundamental group of the fibre $X_{s}$. This approach makes sense for any fundamental group defined algebraically as the Tannaka dual of a category of 'locally constant' coefficients. So if $f: X \rightarrow S$ is a morphism of smooth varieties with section $p$, then $f_{*}: \pi_{1}^{\mathcal{C}_{X}}(X, x) \rightarrow \pi_{1}^{\mathcal{C}_{S}}(S, s)$ is surjective, and $\pi_{1}^{\mathcal{C}_{S}}(S, s)$ acts on the kernel. Here $\mathcal{C}_{(-)}$is any appropriate category of coefficients, for example vector bundles with integrable connection, unipotent isocrystals etc., and e.g. $\pi_{1}^{\mathcal{C}_{X}}(X, x)$ is the Tannaka dual of this category with respect to the fibre functor $x^{*}$. This gives the kernel of $f_{*}$ the structure of an 'affine group scheme over $\mathcal{C}_{S}$ ', and it makes sense to ask what the fibre is over any closed point $s \in S$. The main theorem of the first section is the following.

Theorem. Suppose that $f: X \rightarrow S$ is a smooth morphism of smooth varieties over an algebraically closed field $k$ of characteristic zero. Assume that both $S$ and the fibres of $f$ are connected, and that $X$ is the complement of a relative normal crossings divisor in a smooth and proper $S$-scheme $\bar{X}$. Let $\mathcal{C}_{S}$ be the category of vector bundles with a regular integrable connection on $S$, and let $\mathcal{C}_{X}$ be the category of vector bundles with a regular integrable connection on $X$ which are iterated extensions of those of the form $f^{*} \mathscr{E}$, with $\mathscr{E} \in \mathcal{C}_{S}$. Then the fibre of the corresponding affine group scheme over $\mathcal{C}_{S}$ at $s \in S$ is the de Rham fundamental group $\pi_{1}^{\mathrm{dR}}\left(X_{s}, p_{s}\right)$ of the fibre.

In Section 2 we discuss path torsors in the relative setting. We show in particular that for any other section $q$ of $f$ one can define an affine scheme $\pi_{1}^{\mathrm{dR}}(X / S, q, p)$ over $\mathcal{C}_{S}$ which is a right torsor under the relative de Rham fundamental group 
$\pi_{1}^{\mathrm{dR}}(X / S, p)$. The upshot of this is that we obtain

$$
j_{n}: X(S) \rightarrow H^{1}\left(S, \pi_{1}^{\mathrm{dR}}(X / S, p)_{n}\right)
$$

which are a coarse characteristic zero function field analogue of Kim's global period maps. Of course, if we were really interested in the characteristic zero picture, we would want to define Hodge structures on these objects, and thus obtain finer period maps. However, our main interest lies in the positive characteristic case, and so we don't pursue these questions.

In Section 3 we define the relative rigid fundamental group in positive characteristic, mimicking the definition in characteristic zero. Instead of the category of vector bundles with regular integrable connections, we consider the category of overconvergent $F$-isocrystals (throughout Section 3 we will be over a finite field, and Frobenius will always mean the linear Frobenius). We then proceed to use Caro's theory of cohomological operations for arithmetic $\mathcal{D}$-modules in order to prove the analogue of the above theorem in positive characteristic.

The upshot of this is that for a smooth and proper map $f: X \rightarrow S$ with geometrically connected fibres and smooth, geometrically connected base over a finite field $k$, and a section $p$ of $f$, we can define an affine group scheme $\pi_{1}^{\mathrm{rig}}(X / S, p)$ over the category of overconvergent $F$-isocrystals on $S$, which we call the relative fundamental group at $p$. The fibre of this over any point $s \in S$ is just the unipotent rigid fundamental group of the fibre $X_{s}$ of $f$ over $s$. As in the zero characteristic case, the general Tannakian formalism gives us path torsors $\pi_{1}^{\text {rig }}(X / S, p, q)$ for any other $q \in X(S)$, and hence we can define a period map

$$
X(S) \rightarrow H_{F, \text { rig }}^{1}\left(S, \pi_{1}^{\mathrm{rig}}(X / S, p)\right)
$$

where the RHS is a classifying set of $F$-torsors under $\pi_{1}^{\text {rig }}(X / S, p)$, as well as finite level versions given by pushing out along the quotient map $\pi_{1}^{\mathrm{rig}}(X / S, p) \rightarrow$ $\pi_{1}^{\text {rig }}(X / S, p)_{n}$.

Finally, we study the targets of these period maps, and show that after replacing $H_{F, \text { rig }}^{1}\left(S, \pi_{1}^{\text {rig }}(X / S, p)\right)$, the set classifying $F$-torsors, by the Frobenius invariant part of the set classifying torsors without $F$-structure, $H_{\text {rig }}^{1}\left(S, \pi_{1}^{\text {rig }}(X / S, p)\right)^{\phi=i d}$, then under very restrictive hypotheses on the morphism $f: X \rightarrow S$, we obtain the structure of an algebraic variety. The argument here is just a translation of the original argument of Kim into our context, and what for us are restrictive hypotheses are automatically satisfied in his case.

We are still a long way away from getting a version of Kim's methods to work for function fields. There is still the question of how to define the analogue of the local period maps, and also to show that the domains of the period maps have the structure of varieties. Even then, it is very unclear what the correct analogue of the local integration theory will be in positive characteristic. There is still a very large amount of work to be done if such a project is to be completed. 


\section{Relative de Rham fundamental groups}

Let $f: X \rightarrow S$ be a smooth morphism of smooth complex varieties, and suppose that $f$ admits a good compactification, that is, there exists $\bar{X}$ smooth and proper over $S$, an open immersion $X \hookrightarrow \bar{X}$ over $S$, such that $D=\bar{X} \backslash X$ is a relative normal crossings divisor in $\bar{X}$. Let $p \in X(S)$ be a section. For every closed point $s \in S$ with fibre $X_{s}$, one can consider the topological fundamental group $G_{s}:=\pi_{1}\left(X_{s}^{\text {an }}, p(s)\right)$, and as $s$ varies, these fit together to give a locally constant sheaf $\pi_{1}(X / S, p)$ on $S^{\text {an }}$. Let

$$
\hat{\mathcal{U}}\left(\operatorname{Lie} G_{s}\right):=\lim _{\longleftarrow} \mathrm{C}\left[G_{s}\right] / \mathfrak{a}^{n}
$$

denote the completed enveloping algebra of the Malcev Lie algebra of $G_{s}$, where $\mathfrak{a} \subset \mathbb{C}\left[G_{s}\right]$ is the augmentation ideal. According to Proposition 4.2 of [25], as $s$ varies, these fit together to give a pro-local system on $S^{\text {an }}$, i.e. a pro-object $\hat{\mathcal{U}}_{p}^{\text {top }}$ in the category of locally constant sheaves of finite dimensional C-vector spaces on $S^{\text {an }}$. (Their theorem is a lot stronger than this, but this is all we need for now). According to Théorème 5.9 in Chapter II of [18], the pro-vector bundle with integrable connection $\hat{\mathcal{U}}_{p}^{\text {top }} \otimes_{\mathrm{C}} \mathcal{O}_{S^{\text {an }}}$ has a canonical algebraic structure. Thus given a smooth morphism $f: X \rightarrow S$ as above, with section $p$, one can construct a pro-vector bundle with connection $\hat{\mathcal{U}}_{p}$ on $S$, whose fibre at any closed point $s \in S$ is the completed enveloping algebra of the Malcev Lie algebra of $\pi_{1}\left(X_{s}^{\text {an }}, p(s)\right)$.

Denoting by $\mathfrak{g}_{s}$ the Malcev Lie algebra of $\pi_{1}\left(X_{s}^{\text {an }}, p(s)\right), \hat{\mathcal{U}}\left(\mathfrak{g}_{s}\right)=\left(\hat{\mathcal{U}}_{p}\right)_{s}$ can be constructed algebraically, as $\mathfrak{g}_{s}$ is equal to Lie $\pi_{1}^{\mathrm{dR}}\left(X_{s}, p_{s}\right)$, the Lie algebra of the Tannaka dual of the category of unipotent vector bundles with integrable connection on $X_{s}$. This suggests the question of whether or not there is an algebraic construction of $\hat{\mathcal{U}}_{p}$ ?

We will not directly answer this question - instead we will construct the Lie algebra associated to $\hat{\mathcal{U}}_{p}$ - this is a pro-system $\hat{\mathscr{C}}_{p}$ of Lie algebras with connection on $S$. The way we will do so is very simple, and is closely related to ideas used in [35] to study relatively unipotent mixed motivic sheaves.

DEFINITION 1.1. To save ourselves saying the same thing over and over again, we make the following definition. A 'good' morphism is a smooth morphism $f: X \rightarrow S$ of smooth varieties over a field $k$, with geometrically connected fibres and base, such that $X$ is the complement of a relative normal crossings divisor in a smooth, proper $S$-scheme $\bar{X}$. Throughout this section we will assume that the ground field $k$ is algebraically closed of characteristic 0 .

We will assume that the reader is familiar with Tannakian categories, a good introductory reference is [31]. If $\mathcal{T}$ is a Tannakian category over a field $k$, and $\omega$ is a fibre functor on $\mathcal{T}$, in the sense of $\$ 1.9$ of [20], we will denote the group scheme representing tensor automorphisms of $\omega$ by $G(\mathcal{T}, \omega)$. We will also use the rudiments of algebraic geometry in Tannakian categories, as explained in §5 of [19] - in particular we will talk about affine (group) schemes over Tannakian categories. We 
will denote the fundamental group of a Tannakian category by $\pi(\mathcal{T})$, this is an affine group scheme over $\mathcal{T}$ which satisfies $\omega(\pi(\mathcal{T}))=G(\mathcal{T}, \omega)$ for every fibre functor $\omega$ (see for example 6.1 of [19]). If $\mathcal{T}$ is a Tannakian category over $k$, and $k^{\prime} / k$ is a finite extension, then we will denote the category of $k^{\prime}$-modules in $\mathcal{T}$ by either $\mathcal{T} \otimes_{k} k^{\prime}$, or $\mathcal{T}_{k^{\prime}}$.

We will also assume familiarity with the theory of integrable connections and regular holonomic $\mathcal{D}$-modules on $k$-varieties, and will generally refer to [18] and [23] for details. We say that a regular integrable connection on $X$ is unipotent if it is a successive extension of the trivial connection, and these form a Tannakian subcategory $\mathcal{N} \operatorname{IC}(X) \subset \operatorname{IC}(X)$ of the Tannakian category of regular integrable connections.

Definition 1.2. For $X / k$ smooth and connected, the algebraic and de Rham fundamental groups of $X$ at a closed point $x \in X$ are defined by

$$
\begin{aligned}
& \pi_{1}^{\mathrm{alg}}(X, x):=x^{*}(\pi(\operatorname{IC}(X)))=G\left(\operatorname{IC}(X), x^{*}\right) \\
& \pi_{1}^{\mathrm{dR}}(X, x):=x^{*}(\pi(\mathcal{N} \operatorname{IC}(X)))=G\left(\mathcal{N} \operatorname{IC}(X), x^{*}\right) .
\end{aligned}
$$

REMARK 1.3. It follows from the Riemann-Hilbert correspondence that if $k=\mathrm{C}$, then these affine group schemes are the pro-algebraic and pro-unipotent completions of $\pi_{1}\left(X^{\mathrm{an}}, x\right)$ respectively.

If $f: X \rightarrow Y$ is a morphism of smooth $k$-varieties, then we can form the pullback of vector bundles with integrable connection on $Y$, which preserves regularity and is the usual pull-back on the underlying $\mathcal{O}_{Y}$-module. This induces a homomorphism $f_{*}: \pi_{1}^{\#}(X, x) \rightarrow \pi_{1}^{\#}(Y, f(x))$ for $\#=\mathrm{dR}$, alg.

\section{1 - The relative fundamental group and its pro-nilpotent Lie algebra}

Let $f: X \rightarrow S$ be a 'good' morphism. A regular integrable connection $E$ on $X$ is said to be relatively unipotent if there exists a filtration by horizontal sub-bundles, whose graded objects are all in the essential image of $f^{*}: \operatorname{IC}(S) \rightarrow \operatorname{IC}(X)$. We will denote the full subcategory of relatively unipotent objects in $\operatorname{IC}(X)$ by $\mathcal{N}_{f} \operatorname{IC}(X)$, which is a Tannakian subcategory. Suppose that $p \in X(S)$ is a section of $f$. We have functors of Tannakian categories

$$
\mathcal{N}_{f} \operatorname{IC}(X) \underset{f^{*}}{\stackrel{p^{*}}{\rightleftarrows}} \operatorname{IC}(S)
$$

and hence, after choosing a point $s \in S(k)$, homomorphisms

$$
G\left(\mathcal{N}_{f} \operatorname{IC}(X), p(s)^{*}\right) \underset{p_{*}}{\stackrel{f_{*}}{\rightleftarrows}} G\left(\operatorname{IC}(S), s^{*}\right)
$$


between their Tannaka duals. Let $K_{s}$ denote the kernel of $f_{*}$. Then the splitting $p_{*}$ induces an action of $\pi_{1}^{\mathrm{alg}}(S, s)=G\left(\operatorname{IC}(S), s^{*}\right)$ on $K_{s}$ via conjugation. This corresponds to an affine group scheme over $\operatorname{IC}(S)$.

LEMma 1.4. This affine group scheme over $\operatorname{IC}(S)$ is independent of $s$.

Proof. Thanks to [19], §6.4, $f_{*}, p_{*}$ above come from homomorphisms

$$
p^{*}\left(\pi\left(\mathcal{N}_{f} \mathrm{IC}(X)\right)\right) \underset{p_{*}}{\stackrel{f_{*}}{\rightleftarrows}} \pi(\mathrm{IC}(S))
$$

of affine group schemes over $\operatorname{IC}(S)$. If we let $\mathcal{K}$ denote the kernel of $f_{*}$, then $K_{s}=s^{*}(\mathcal{K})$, and this induces the action of $\pi_{1}^{\text {alg }}(S, s)$ on $K_{s}$.

Definition 1.5. The relative de Rham fundamental group $\pi_{1}^{\mathrm{dR}}(X / S, p)$ of $X / S$ at $p$ is defined to be the affine group scheme $\mathcal{K}$ over $\operatorname{IC}(S)$.

Let $i_{s}: X_{s} \rightarrow X$ denote the inclusion of the fibre over $s$. Then there is a canonical functor $i_{s}^{*}: \mathcal{N}_{f} \operatorname{IC}(X) \rightarrow \mathcal{N} \operatorname{IC}\left(X_{s}\right)$. This induces a homomorphism $\pi_{1}^{\mathrm{dR}}\left(X_{s}, p_{s}\right) \rightarrow$ $G\left(\mathcal{N}_{f} \mathrm{IC}(X), p_{s}^{*}\right)$ which is easily seen to factor through the fibre $\pi_{1}^{\mathrm{dR}}(X / S, p)_{s}:=$ $s^{*}(\mathcal{K})=K_{s}$ of $\pi_{1}^{\mathrm{dR}}(X / S, p)$ over $s$.

Theorem 1.6. Suppose that $k=$ C. Then $\phi: \pi_{1}^{\mathrm{dR}}\left(X_{s}, p_{s}\right) \rightarrow \pi_{1}^{\mathrm{dR}}(X / S, p)_{s}$ is an isomorphism.

Proof. The point $s$ gives us fibre functors $p_{s}^{*}$ on $\mathcal{N} \operatorname{IC}\left(X_{s}\right), p(s)^{*}$ on $\mathcal{N}_{f} \operatorname{IC}(X)$ and $s^{*}$ on $\operatorname{IC}(S)$. Write

$$
\mathcal{K}=G\left(\mathcal{N} \operatorname{IC}\left(X_{s}\right), p_{s}^{*}\right), \quad \mathcal{G}=G\left(\mathcal{N}_{f} \operatorname{IC}(X), p(s)^{*}\right), \quad \mathcal{H}=G\left(\operatorname{IC}(S), s^{*}\right)
$$

and also let

$$
K=\pi_{1}\left(X_{s}^{\mathrm{an}}, p(s)\right), \quad G=\pi_{1}\left(X^{\mathrm{an}}, p(s)\right), \quad H=\pi_{1}\left(S^{\mathrm{an}}, s\right)
$$

be the topological fundamental groups of $X_{s}, X, S$ respectively. Then $\mathcal{K}=K^{\text {un }}$, the pro-unipotent completion of $K$, and $\mathcal{H}=H^{\text {alg }}$, the pro-algebraic completion of $H$. We need to show that the sequence of affine group schemes

$$
1 \rightarrow \mathcal{K} \rightarrow \mathcal{G} \rightarrow \mathcal{H} \rightarrow 1
$$

is exact, and we will use the equivalences of categories

$$
\begin{gathered}
\operatorname{IC}(X) \stackrel{\sim}{\rightarrow} \operatorname{Rep}_{\mathrm{C}}\left(\pi_{1}\left(X^{\mathrm{an}}, p(s)\right)\right), \quad \operatorname{IC}(S) \stackrel{\sim}{\rightarrow} \operatorname{Rep}_{\mathrm{C}}\left(\pi_{1}\left(S^{\mathrm{an}}, s\right)\right) \\
\operatorname{IC}\left(X_{s}\right) \stackrel{\sim}{\rightarrow} \operatorname{Rep}_{\mathrm{C}}\left(\pi_{1}\left(X_{s}^{\mathrm{an}}, p(s)\right)\right) .
\end{gathered}
$$

By Proposition 1.3 in Chapter I of [35], $\operatorname{ker}(\mathcal{G} \rightarrow \mathcal{H})$ is pro-unipotent. Hence according to Proposition 1.4 of loc. cit., in order to show that $\phi$ is an isomorphism, we must show the following. 
- If $E \in \mathcal{N}_{f} \mathrm{IC}(X)$ is such that $i_{s}^{*}(E)$ is trivial, then $E \cong f^{*}(F)$ for some $F$ in $\operatorname{IC}(S)$.

- Let $E \in \mathcal{N}_{f} \mathrm{IC}(X)$, and let $F_{0} \subset i_{s}^{*}(E)$ denote the largest trivial sub-object. Then there exists $E_{0} \subset E$ such that $F_{0}=i_{s}^{*}\left(E_{0}\right)$.

- There is a pro-action of $\mathcal{G}$ on $\hat{\mathcal{U}}($ Lie $\mathcal{K})$ such that the corresponding action of Lie $\mathcal{G}$ extends the left multiplication by Lie $\mathcal{K}$.

The first is straightforward. Since $f$ is topologically a fibration with section $p$, we have a split exact sequence

$$
1 \rightarrow K \rightarrow G \leftrightarrows H \rightarrow 1
$$

and a representation $V$ of $G$ such that $K$ acts trivially. We must show that $V$ is the pullback of an $H$-representation - this is obvious! The second is no harder, we must show that if $V$ is a $G$-representation, then $V^{K}$ is a sub- $G$-module of $V$. But since $K$ is normal in $G$, this is clear. For the third, note that $\hat{\mathcal{U}}($ Lie $\mathcal{K})=\hat{\mathcal{U}}($ Lie $K)=$ $\lim \mathrm{C}[K] / \mathfrak{a}^{n}$, where $\mathfrak{a}$ is the augmentation ideal of $\mathrm{C}[K]$. Let $H$ act on $\mathrm{C}[K] / \mathfrak{a}^{n}$ by

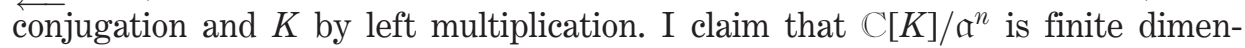
sional, and unipotent as a $K$-representation.

Indeed, there are extensions of $K$-representations

$$
0 \rightarrow \mathfrak{a}^{n} / \mathfrak{a}^{n+1} \rightarrow \mathrm{C}[K] / \mathfrak{a}^{n+1} \rightarrow \mathrm{C}[K] / \mathfrak{a}^{n} \rightarrow 0
$$

and hence, since the action of $K$ on $\mathfrak{a}^{n} / \mathfrak{a}^{n+1}$ is trivial, it follows by induction that each $\mathrm{C}[K] / \mathfrak{a}^{n}$ is unipotent. There are also surjections

$$
\left(\mathfrak{a} / \mathfrak{a}^{2}\right)^{\otimes n} \rightarrow \mathfrak{a}^{n} / \mathfrak{a}^{n+1}
$$

for each $n$, and hence by induction, to show finite dimensionality it suffices to show that $\mathfrak{a} / \mathfrak{a}^{2}$ is finite dimensional. But $\mathfrak{a} / \mathfrak{a}^{2} \cong K^{\mathrm{ab}} \otimes_{\mathrm{Z}} \mathrm{C}$ is finite dimensional, as $K$ is finitely generated.

Now, since $\mathrm{C}[K] / \mathfrak{a}^{n}$ is unipotent as a $K$-representation, it is relatively unipotent as a $G=K \times H$-representation, hence $\mathrm{C}[K] / \mathfrak{a}^{n}$ is naturally an object in $\operatorname{Rep}_{\mathbb{C}}(\mathcal{G})$. Thus there is a pro-action of $\mathcal{G}$ on $\hat{\mathcal{U}}$ (Lie $\mathcal{K})$, and the action extends left multiplication by Lie $\mathcal{K}$ as required.

REMARK 1.7. The co-ordinate algebra of $\pi_{1}^{\mathrm{dR}}(X / S, p)$ is an ind-object in the category of regular integrable connections on $S$. Hence we may view $\pi_{1}^{\mathrm{dR}}(X / S, p)$ as an affine group scheme over $S$ in the usual sense, together with a regular integrable connection on the associated $\mathcal{O}_{S}$-Hopf algebra.

If $g: T \rightarrow S$ is any morphism of smooth varieties over $k$, then there is a homomorphism of fundamental groups

$$
\pi_{1}^{\mathrm{dR}}\left(X_{T} / T, p_{T}\right) \rightarrow \pi_{1}^{\mathrm{dR}}(X / S, p) \times_{S} T:=g^{*}\left(\pi_{1}^{\mathrm{dR}}(X / S, p)\right)
$$


which corresponds to a horizontal morphism

$$
\mathcal{O}_{\pi_{1}^{\mathrm{dR}}(X / S, p)} \otimes_{\mathcal{O}_{S}} \mathcal{O}_{T} \rightarrow \mathcal{O}_{\pi_{1}^{\mathrm{dR}}\left(X_{T} / T, p_{T}\right)} .
$$

Proposition 1.8. If $k=\mathrm{C}$ then this is an isomorphism.

Proof. We know by the previous theorem that this induces an isomorphism on fibres over any point $t \in T(\mathbb{C})$. Hence by rigidity, it is an isomorphism.

Write $G=\pi_{1}^{\mathrm{dR}}(X / S, p)$ and let $G_{n}$ denote the quotient of $G$ by the $n$th term in its lower central series. Let $A_{n}$ denote the Hopf algebra of $G_{n}$, and $I_{n} \subset A_{n}$ the augmentation ideal. $L_{n}:=\mathcal{H} \mathrm{m}_{\mathcal{O}_{S}}\left(I_{n} / I_{n}^{2}, \mathcal{O}_{S}\right)$ is the Lie algebra of $G_{n}$. This is a coherent, nilpotent Lie algebra with connection, i.e the bracket [., . ] $: L_{n} \otimes L_{n} \rightarrow L_{n}$ is horizontal. There are natural morphisms $L_{n+1} \rightarrow L_{n}$, which form a pro-system of nilpotent Lie algebras with connection $\hat{L}_{p}$, whose universal enveloping algebra is the object $\hat{\mathcal{U}}_{p}$ considered in the introduction to this section.

\section{2 - An algebraic proof of Theorem 1.6}

Although we have a candidate for the relative fundamental group of a 'good' morphism $f: X \rightarrow S$ at a section $p$, so far we have only proved it is a good candidate when the ground field is the complex numbers. One might hope to be able to reduce to the case $k=\mathrm{C}$ via base change and finiteness arguments, but this approach will not work in a straightforward manner. Also, such an argument will not easily adapt to the case of positive characteristic, as in general one will not be able to lift a smooth proper family, even locally on the base. In this section, we will give a more algebraic proof, that will more easily adapt to positive characteristic. Recall that we have an affine group scheme $\pi_{1}^{\mathrm{dR}}(X / S, p)$ over $\mathrm{IC}(S)$, and a comparison morphism

$$
\phi: \pi_{1}^{\mathrm{dR}}\left(X_{s}, p_{s}\right) \rightarrow \pi_{1}^{\mathrm{dR}}(X / S, p)_{s}
$$

for any point $s \in S$. We will prove that this map is an isomorphism.

It follows from Proposition 1.4 in Chapter I of [35] and Appendix A of [22] that we need to prove the following:

- (Injectivity) Every $E \in \mathcal{N} \operatorname{IC}\left(X_{s}\right)$ is a quotient of $i_{s}^{*}(F)$ for some $F \in \mathcal{N}_{f} \operatorname{IC}(X)$.

- (Surjectivity I) Suppose that $E \in \mathcal{N}_{f} \mathrm{IC}(X)$ is such that $i_{s}^{*}(E)$ is trivial. Then there exists $F \in \operatorname{IC}(S)$ such that $E \cong f^{*}(F)$.

- (Surjectivity II) Let $E \in \mathcal{N}_{f} \mathrm{IC}(X)$, and let $F_{0} \subset i_{s}^{*}(E)$ denote the largest trivial sub-object. Then there exists $E_{0} \subset E$ such that $F_{0}=i_{s}^{*}\left(E_{0}\right)$.

To do so, we will need to use the language of algebraic $\mathcal{D}$-modules. We define the functor

$$
f_{*}^{\mathrm{dR}}: \mathcal{N}_{f} \mathrm{IC}(X) \rightarrow \mathrm{IC}(S)
$$


by $f_{*}^{\mathrm{dR}}(E)=\mathcal{H}^{-d}\left(f_{+} E\right)$ where $f_{+}$is the usual push-forward for regular holonomic complexes of $\mathscr{D}$-modules, $d$ is the relative dimension of $f: X \rightarrow S$, and we are considering a regular integrable connection on $X$ as a $\mathcal{D}_{X}$-module in the usual way.

LEMMA 1.9. The functor $f_{*}^{\mathrm{dR}}$ lands in the category of regular integrable connections, and is a right adjoint to $f^{*}$.

Proof. The content of the first claim is in the coherence of direct images in de Rham cohomology, using the comparison result 1.4 of [21], and the fact that a regular holonomic $\mathcal{D}_{X}$-module is a vector bundle iff it is coherent as an $\mathcal{O}_{X}$-module.

To see this coherence, we first use adjointness of $f_{+}$and $f^{+}$, together with the facts that $f^{+} \mathcal{O}_{S}=\mathcal{O}_{X}[-d]$ and $f_{+} \mathcal{O}_{X}$ is concentrated in degrees $\geq-d$, to get canonical adjunction morphism $f_{*}^{\mathrm{dR}}\left(\mathcal{O}_{X}\right) \rightarrow \mathcal{O}_{S}$ of regular holonomic $\mathcal{D}_{X}$-modules. I claim that this is an isomorphism. Indeed, by 1.4 of [21] we have $f_{*}^{\mathrm{dR}}\left(\mathcal{O}_{X}\right) \cong$ $\mathbf{R}^{0} f_{*}\left(\Omega_{X / S}^{*}\right)$, and since $f$ admits a good compactification $\bar{f}: \bar{X} \rightarrow S$ the latter is isomorphic to $\mathbf{R}^{0} \bar{f}_{*}\left(\Omega_{\bar{X} / S}^{*}(\log D)\right)$ where $D=\bar{X} \backslash X$. Since $\bar{f}$ is proper with connected

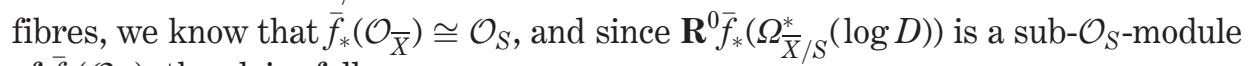
of $\bar{f}_{*}\left(\mathcal{O}_{\bar{X}}\right)$, the claim follows.

Hence in particular $f_{*}^{\mathrm{dR}}\left(\mathcal{O}_{X}\right)$ is coherent, and via the projection formula, so is $f_{*}^{\mathrm{dR}}\left(f^{*} F\right)$ for any $F \in \operatorname{IC}(S)$. Hence using exact sequences in cohomology and induction on unipotence degree, $f_{*}^{\mathrm{dR}} E$ is coherent whenever $E$ is relatively unipotent.

To prove to the second claim, we just use that $f^{+}$is adjoint to $f_{+}, f^{+}=f^{*}[-d]$ on the subcategory of regular integrable connections, and $f_{+} E$ is concentrated in degrees $\geq-d$ whenever $E$ is a regular integrable connection.

REMARK 1.10. Although the proposition is stated in [21] for $k=\mathrm{C}$, the same proof works for any algebraically closed field of characteristic zero.

Thus we get a canonical morphism $\varepsilon_{E}: f^{*} f_{*}^{\mathrm{dR}} E \rightarrow E$ which is the counit of the adjunction between $f^{*}$ and $f_{*}^{\mathrm{dR}}$.

Example 1.11. Suppose that $S=\operatorname{Spec}(k)$. Then

$$
f_{*}^{\mathrm{dR}} E=H_{\mathrm{dR}}^{0}(X, E)=\operatorname{Hom}_{\mathcal{N} \operatorname{IC}(X)}\left(\mathcal{O}_{X}, E\right)
$$

and the adjunction becomes the identification

$$
\operatorname{Hom}_{\mathcal{N} \operatorname{IC}(X)}\left(V \otimes_{k} \mathcal{O}_{X}, E\right)=\operatorname{Hom}_{\operatorname{Vec}_{k}}\left(V, \operatorname{Hom}_{\mathcal{N I C}(X)}\left(\mathcal{O}_{X}, E\right)\right) .
$$

Since $f_{*}^{\mathrm{dR}}$ takes objects in $\mathcal{N}_{f} \mathrm{IC}(X)$ to objects in $\mathrm{IC}(S)$, it commutes with base change and there is an isomorphism of functors

$$
H_{\mathrm{dR}}^{0}\left(X_{s},-\right) \circ i_{s}^{*} \cong s^{*} \circ f_{*}^{\mathrm{dR}}: \mathcal{N}_{f} \mathrm{IC}(X) \rightarrow \operatorname{Vec}_{k} .
$$

To see this, first note that by using the five lemma, the projection formula and 
induction on the relative unipotence degree, it suffices to prove that $f_{*}^{\mathrm{dR}}\left(\mathcal{O}_{X}\right)$ and $\mathbf{R}_{\mathrm{dR}}^{1} f_{*}\left(\mathcal{O}_{X}\right)$ commute with base change, where we have written $\mathbf{R}_{\mathrm{dR}}^{1} f_{*}\left(\mathcal{O}_{X}\right)=$ $\mathcal{H}^{-d+1}\left(f_{+} \mathcal{O}_{X}\right)$. Again, using 1.4 of [21] this boils down to proving that $\mathbf{R}^{i} f_{*}\left(\Omega_{X / S}^{*}\right)$ commute with base change for $i=0,1$. Since we know that they are coherent (and hence locally free), and that $f: X \rightarrow S$ admits a good compactification $\bar{f}: \bar{X} \rightarrow S$, this follows from Corollary 8.6 of [27] together with the standard isomorphism $\left.\mathbf{R}^{i} f_{*}\left(\Omega_{X / S}^{*}\right) \cong \mathbf{R}_{f_{*}^{i}} \bar{f}_{\bar{X} / S}(\log D)\right)$ where $D=\bar{X} \backslash X$.

Proposition 1.12. Suppose that $i_{s}^{*} E$ is trivial. Then the counit $\varepsilon_{E}: f^{*} f_{*}^{\mathrm{dR}} E \rightarrow E$ is an isomorphism.

Proof. Pulling back $\varepsilon_{E}$ by $i_{s}^{*}$, and using base change, we get a morphism

$$
\mathcal{O}_{X_{s}} \otimes_{k} H_{\mathrm{dR}}^{0}\left(X_{s}, i_{s}^{*} E\right) \rightarrow i_{s}^{*} E
$$

which by the explicit description of 1.11 is seen to be an isomorphism (as $i_{s}^{*} E$ is trivial). Hence by rigidity, $\varepsilon_{E}$ must be an isomorphism.

Proposition 1.13. Let $E \in \mathcal{N}_{f} \mathrm{IC}(X)$, and let $F_{0} \subset i_{s}^{*}(E)$ denote the largest trivial sub-object. Then there exists $E_{0} \subset E$ such that $F_{0}=i_{s}^{*}\left(E_{0}\right)$.

Proof. Let $F=i_{s}^{*}(E)$, then we have $H_{\mathrm{dR}}^{0}\left(X_{s}, F\right)=\operatorname{Hom}_{\mathrm{IC}\left(X_{s}\right)}\left(\mathcal{O}_{X_{s}}, F\right)$. I first claim that the natural map $\mathcal{O}_{X_{s}} \otimes_{k} H_{\mathrm{dR}}^{0}\left(X_{s}, F\right) \rightarrow F$ is injective and identifies $F_{0}$ with $\mathcal{O}_{X_{s}} \otimes_{k} H_{\mathrm{dR}}^{0}\left(X_{s}, F\right)$. But since the category $\mathcal{N} \operatorname{IC}\left(X_{s}\right)$ is Tannakian, this is equivalent to claiming that for an affine group scheme $G$ over $k$, and $V$ a representation of $G$, the natural map of $G$ representations $H^{0}(G, V) \rightarrow V$ is injective, and identifies $H^{0}(G, V)$ with the largest trivial sub- $G$-module of $V$. This is obvious.

To complete the proof, set $E_{0}=f^{*} f_{*}^{\mathrm{dR}}(E)$, then by the base change results we know that $i_{s}^{*}\left(E_{0}\right) \cong F_{0}$, and that the natural map $E_{0} \rightarrow E$ restricts to the inclusion $F_{0} \rightarrow F$ on the fibre $X_{s}$.

Corollary 1.14. The map $\pi_{1}^{\mathrm{dR}}\left(X_{s}, p_{s}\right) \rightarrow \pi_{1}^{\mathrm{dR}}(X / S, p)_{s}$ is a surjection.

We now turn to the proof of injectivity of the comparison map, borrowing heavily from ideas used in Section 2.1 of [24]. We define objects $U_{n}$ of $\mathcal{N} \operatorname{IC}\left(X_{s}\right)$, the category of unipotent integrable connections on $X_{s}$ inductively as follows. $U_{1}$ will just be $\mathcal{O}_{X_{s}}$, and $U_{n+1}$ will be the extension of $U_{n}$ by $\mathcal{O}_{X_{s}} \otimes_{k} H_{\mathrm{dR}}^{1}\left(X_{s}, U_{n}^{\vee}\right)^{\vee}$ corresponding to the identity under the isomorphisms

$$
\begin{aligned}
\operatorname{Ext}_{\mathrm{IC}\left(X_{s}\right)}\left(U_{n}, \mathcal{O}_{X_{s}} \otimes_{k} H_{\mathrm{dR}}^{1}\left(X_{s}, U_{n}^{\vee}\right)^{\vee}\right) & \cong H_{\mathrm{dR}}^{1}\left(X_{s}, U_{n}^{\vee} \otimes_{k} H_{\mathrm{dR}}^{1}\left(X_{s}, U_{n}^{\vee}\right)^{\vee}\right) \\
& \cong H_{\mathrm{dR}}^{1}\left(X_{s}, U_{n}^{\vee}\right) \otimes_{k} H_{\mathrm{dR}}^{1}\left(X_{s}, U_{n}^{\vee}\right)^{\vee} \\
& \cong \operatorname{End}_{k}\left(H_{\mathrm{dR}}^{1}\left(X_{s}, U_{n}^{\vee}\right)\right) .
\end{aligned}
$$


If we look at the long exact sequence in de Rham cohomology associated to the short exact sequence $0 \rightarrow U_{n}^{\vee} \rightarrow U_{n+1}^{\vee} \rightarrow H_{\mathrm{dR}}^{1}\left(X_{s}, U_{n}^{\vee}\right) \otimes_{k} \mathcal{O}_{X_{s}} \rightarrow 0$ we get

$$
\begin{aligned}
0 \rightarrow H_{\mathrm{dR}}^{0}\left(X_{s}, U_{n}^{\vee}\right) & \rightarrow H_{\mathrm{dR}}^{0}\left(X_{s}, U_{n+1}^{\vee}\right) \rightarrow H_{\mathrm{dR}}^{1}\left(X_{s}, U_{n}^{\vee}\right) \\
& \stackrel{\delta}{\rightarrow} H_{\mathrm{dR}}^{1}\left(X_{s}, U_{n}^{\vee}\right) \rightarrow H_{\mathrm{dR}}^{1}\left(X_{s}, U_{n+1}^{\vee}\right) .
\end{aligned}
$$

LEMMA 1.15. The connecting homomorphism $\delta$ is the identity.

Proof. By dualising, the extension

$$
0 \rightarrow U_{n}^{\vee} \rightarrow U_{n+1}^{\vee} \rightarrow \mathcal{O}_{X_{s}} \otimes_{k} H_{\mathrm{dR}}^{1}\left(X_{s}, U_{n}^{\vee}\right) \rightarrow 0
$$

corresponds to the identity under the isomorphism

$$
\operatorname{Ext}_{\mathrm{IC}\left(X_{s}\right)}\left(\mathcal{O}_{X_{s}} \otimes_{k} H_{\mathrm{dR}}^{1}\left(X_{s}, U_{n}^{\vee}\right), U_{n}^{\vee}\right) \cong \operatorname{End}_{k}\left(H_{\mathrm{dR}}^{1}\left(X_{s}, U_{n}^{\vee}\right)\right) .
$$

Now the lemma follows from the fact that for an extension $0 \rightarrow E \rightarrow$ $F \rightarrow \mathcal{O}_{X_{s}} \otimes_{k} V \rightarrow 0$ of a trivial bundle by $E$, the class of the extension under the isomorphism

$$
\operatorname{Ext}_{\mathrm{IC}\left(X_{s}\right)}\left(\mathcal{O}_{X_{s}} \otimes_{k} V, E\right) \cong V^{\vee} \otimes H_{\mathrm{dR}}^{1}\left(X_{s}, E\right) \cong \operatorname{Hom}_{k}\left(V, H_{\mathrm{dR}}^{1}\left(X_{s}, E\right)\right)
$$

is just the connecting homomorphism for the long exact sequence

$$
0 \rightarrow H_{\mathrm{dR}}^{0}\left(X_{s}, E\right) \rightarrow H_{\mathrm{dR}}^{0}\left(X_{s}, F\right) \rightarrow V \rightarrow H_{\mathrm{dR}}^{1}\left(X_{s}, E\right) .
$$

In particular, any extension of $U_{n}$ by a trivial bundle $V \otimes_{k} \mathcal{O}_{X_{s}}$ is split after pulling back to $U_{n+1}$, and $H_{\mathrm{dR}}^{0}\left(X_{s}, U_{n+1}^{\vee}\right) \cong H_{\mathrm{dR}}^{0}\left(X_{s}, U_{n}^{\vee}\right)$. It then follows by induction that $H_{\mathrm{dR}}^{0}\left(X_{s}, U_{n}^{\vee}\right) \cong H_{\mathrm{dR}}^{0}\left(X_{s}, \mathcal{O}_{X_{s}}\right) \cong k$ for all $n$.

Definition 1.16. We define the unipotent class of an object $E \in \mathcal{N} \operatorname{IC}\left(X_{s}\right)$ inductively as follows. If $E$ is trivial, then we say $E$ has unipotent class 1 . If there exists an extension

$$
0 \rightarrow V \otimes_{k} \mathcal{O}_{X_{s}} \rightarrow E \rightarrow E^{\prime} \rightarrow 0
$$

with $E^{\prime}$ of unipotent class $\leq m-1$, then we say that $E$ has unipotent class $\leq m$.

Now let $x=p(s), u_{1}=1 \in\left(U_{1}\right)_{x} \cong k$, and choose a compatible system of elements $u_{n} \in\left(U_{n}\right)_{x}$ mapping to $u_{1}$.

Proposition 1.17. Let $F \in \mathcal{N} \operatorname{IC}\left(X_{s}\right)$ be an object of unipotent class $\leq m$. Then for all $n \geq m$ and any $f \in F_{x}$ there exists a morphism $\alpha: U_{n} \rightarrow F$ such that $\alpha_{x}\left(u_{n}\right)=f$.

Proof. We copy the proof of Proposition 2.1.6 of [24] and use induction on $m$. The case $m=1$ is straightforward. For the inductive step, let $F$ be of unipotent 
class $m$, and choose an exact sequence

$$
0 \rightarrow E \stackrel{\psi}{\rightarrow} E \stackrel{\phi}{\rightarrow} G \rightarrow 0
$$

with $E$ trivial and $G$ of unipotent class $<m$. By induction there exists a morphism $\beta: U_{n-1} \rightarrow G$ such that $\phi_{x}(f)=\beta_{x}\left(u_{n-1}\right)$. Pulling back the extension (33) first by the morphism $\beta$ and then by the natural surjection $U_{n} \rightarrow U_{n-1}$ gives an extension of $U_{n}$ by $E$, which must split, as observed above.

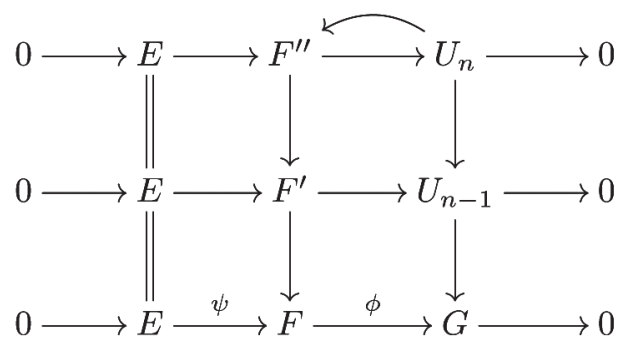

Let $\gamma: U_{n} \rightarrow F$ denote the induced morphism, then $\phi_{x}\left(\gamma_{x}\left(u_{n}\right)-f\right)=0$. Hence there exists some $e \in E_{x}$ such that $\psi_{x}(e)=\gamma_{x}\left(u_{n}\right)-f$. Again by induction we can choose $\gamma^{\prime}: U_{n} \rightarrow E$ with $\gamma_{x}^{\prime}\left(u_{n}\right)=e$. Finally let $\alpha=\gamma-\psi \circ \gamma^{\prime}$, it is easily seen that $\alpha_{x}\left(u_{n}\right)=f$.

Corollary 1.18. Every $E$ in $\mathcal{N} \mathrm{IC}\left(X_{s}\right)$ is a quotient of $U_{m}^{\oplus N}$ for some $m, N \in \mathbb{N}$.

Proof. Suppose that $E$ is of unipotent class $\leq m$. Let $e_{1}, \ldots, e_{N}$ be a basis for $E_{x}$. Then there is a morphism $\alpha: U_{m}^{\oplus N} \rightarrow E$ with every $e_{i}$ in the image of the induced map on fibres. Thus $\alpha_{x}$ is surjective, and hence so is $\alpha$.

We now try to inductively define relatively nilpotent integrable connections $W_{n}$. on $X$ which restrict to the $U_{n}$ on fibres. Define higher direct images in de Rham cohomology by $\mathbf{R}_{\mathrm{dR}}^{i} f_{*}(E)=\mathcal{H}^{i-d}\left(f_{+} E\right)$, and begin the induction with $W_{1}=\mathcal{O}_{X}$. As part of the induction we will assume that $\mathbf{R}_{\mathrm{dR}}^{0} f_{*}\left(W_{n}^{\vee}\right) \cong \mathbf{R}_{\mathrm{dR}}^{0} f_{*}\left(\mathcal{O}_{X}\right)=\mathcal{O}_{S}$, that $\mathbf{R}_{\mathrm{dR}}^{1} f_{*}\left(W^{\vee}\right)$ and $\mathbf{R}_{\mathrm{dR}}^{1} f_{*}(W)$ are both coherent, i.e. regular integrable connections, and that there exists a morphism $p^{*} W_{n}^{\vee} \rightarrow \mathcal{O}_{S}$ such that the composite $\mathcal{O}_{S} \cong f_{*} W_{n}^{\vee} \cong p^{*} f^{*} f_{*} W_{n}^{\vee} \rightarrow p^{*} W_{n}^{\vee} \rightarrow \mathcal{O}_{S}$ is an isomorphism. We will define $W_{n+1}$ to be an extension of $W_{n}$ by the sheaf $f^{*} \mathbf{R}_{\mathrm{dR}}^{1} f_{*}\left(W_{n}^{\vee}\right)^{\vee}$, and thus consider the extension group

$$
\operatorname{Ext}_{\mathrm{IC}(X)}\left(W_{n}, f^{*} \mathbf{R}_{\mathrm{dR}}^{1} f_{*}\left(W_{n}^{\vee}\right)^{\vee}\right) \cong H_{\mathrm{dR}}^{1}\left(X, W_{n}^{\vee} \otimes_{\mathcal{O}_{X}} f^{*} \mathbf{R}_{\mathrm{dR}}^{1} f_{*}\left(W_{n}^{\vee}\right)^{\vee}\right)
$$

The Leray spectral sequence, together with the induction hypothesis and the projection formula, gives us the 5-term exact sequence 
(36)

$$
\begin{aligned}
0 & \rightarrow H_{\mathrm{dR}}^{1}\left(S, \mathbf{R}_{\mathrm{dR}}^{1} f_{*}\left(W_{n}^{\vee}\right)^{\vee}\right) \rightarrow \operatorname{Ext}_{\mathrm{IC}(X)}\left(W_{n}, f^{*} \mathbf{R}_{\mathrm{dR}}^{1} f_{*}\left(W_{n}^{\vee}\right)^{\vee}\right) \\
& \rightarrow \operatorname{End}_{\mathrm{IC}(S)}\left(\mathbf{R}_{\mathrm{dR}}^{1} f_{*}\left(W_{n}^{\vee}\right)\right) \rightarrow H_{\mathrm{dR}}^{2}\left(S, \mathbf{R}_{\mathrm{dR}}^{1} f_{*}\left(W_{n}^{\vee}\right)^{\vee}\right) \\
& \rightarrow H_{\mathrm{dR}}^{2}\left(X, W_{n}^{\vee} \otimes_{\mathcal{O}_{X}}\left(\mathbf{R}_{\mathrm{dR}}^{1} f_{*} W_{n}^{\vee}\right)^{\vee}\right) .
\end{aligned}
$$

Now, the projection $p^{*} W_{n}^{\vee} \rightarrow \mathcal{O}_{S}$ induces a map

$$
H_{\mathrm{dR}}^{i}\left(S, p^{*} W_{n}^{\vee} \otimes_{\mathcal{O}_{X}}\left(\mathbf{R}_{\mathrm{dR}}^{1} f_{*} W_{n}^{\vee}\right)^{\vee}\right) \rightarrow H_{\mathrm{dR}}^{i}\left(S, \mathbf{R}_{\mathrm{dR}}^{1} f_{*}\left(W_{n}^{\vee}\right)^{\vee}\right)
$$

such that the composite (dotted) arrow

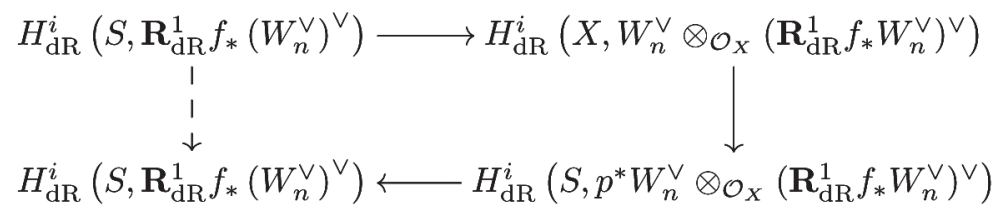

is as isomorphism, since it can be identified with the map induced by the composite arrow $\mathcal{O}_{S} \cong f_{*} W_{n}^{\vee} \cong p^{*} f^{*} f_{*} W_{n}^{\vee} \rightarrow p^{*} W_{n}^{\vee} \rightarrow \mathcal{O}_{S}$. Hence both the maps

$$
\begin{aligned}
& H_{\mathrm{dR}}^{1}\left(S, \mathbf{R}_{\mathrm{dR}}^{1} f_{*}\left(W_{n}^{\vee}\right)^{\vee}\right) \rightarrow \operatorname{Ext}_{\mathrm{IC}(X)}\left(W_{n}, f^{*} \mathbf{R}_{\mathrm{dR}}^{1} f_{*}\left(W_{n}^{\vee}\right)^{\vee}\right) \\
& H_{\mathrm{dR}}^{2}\left(S, \mathbf{R}_{\mathrm{dR}}^{1} f_{*}\left(W_{n}^{\vee}\right)^{\vee}\right) \rightarrow H_{\mathrm{dR}}^{2}\left(X, W_{n}^{\vee} \otimes_{\mathcal{O}_{X}}\left(\mathbf{R}_{\mathrm{dR}}^{1} f_{*} W_{n}^{\vee}\right)^{\vee}\right)
\end{aligned}
$$

appearing in the 5-term exact sequence split. So there is a commutative diagram

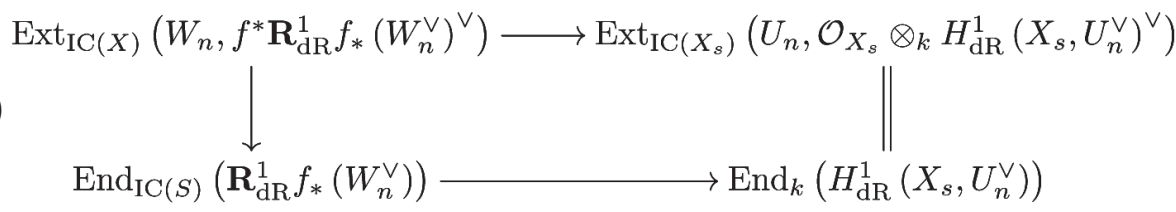

where the horizontal arrows are just restrictions to fibres, and the left hand vertical arrow is surjective. The identity morphism in $\operatorname{End}_{k}\left(H_{\mathrm{dR}}^{1}\left(X_{s}, U_{n}^{\vee}\right)\right)$ clearly lifts to $\operatorname{End}_{\mathrm{IC}(S)}\left(\mathbf{R}_{\mathrm{dR}}^{1} f_{*}\left(W_{n}^{\vee}\right)\right)$, and there exists a unique element of the extension group $\operatorname{Ext}_{\mathrm{IC}(X)}\left(W_{n}, f^{*} \mathbf{R}_{\mathrm{dR}}^{1} f_{*}\left(W_{n}^{\vee}\right)^{\vee}\right)$ lifting the identity in $\operatorname{End}_{\mathrm{IC}(S)}\left(\mathbf{R}_{\mathrm{dR}}^{1} f_{*}\left(W_{n}^{\vee}\right)\right)$, and which maps to zero under the above splitting

$$
\operatorname{Ext}_{\mathrm{IC}(X)}\left(W_{n}, f^{*} \mathbf{R}_{\mathrm{dR}}^{1} f_{*}\left(W_{n}^{\vee}\right)^{\vee}\right) \rightarrow H_{\mathrm{dR}}^{1}\left(S, \mathbf{R}_{\mathrm{dR}}^{1} f_{*}\left(W_{n}^{\vee}\right)^{\vee}\right) .
$$

Let $W_{n+1}$ be the corresponding extension.

Proposition 1.19. Every object of $\mathcal{N} \mathrm{IC}\left(X_{s}\right)$ is a quotient of $i_{s}^{*} E$ for some $E \in \mathcal{N}_{f} \mathrm{IC}(X)$. 
Proof. To finish the induction step, we must show that

$$
\mathbf{R}_{\mathrm{dR}}^{0} f_{*}\left(W_{n+1}^{\vee}\right) \cong \mathbf{R}_{\mathrm{dR}}^{0} f_{*}\left(W_{n}^{\vee}\right),
$$

that $\mathbf{R}_{\mathrm{dR}}^{1} f_{*}\left(W_{n+1}^{\vee}\right)$ and $\mathbf{R}_{\mathrm{dR}}^{1} f_{*}\left(W_{n+1}\right)$ are coherent, and that there exists a morphism $p^{*} W_{n+1}^{\vee} \rightarrow \mathcal{O}_{S}$ as in the induction hypothesis. For the first claim, if we look at the long exact sequence of relative de Rham cohomology

$$
0 \rightarrow \mathbf{R}_{\mathrm{dR}}^{0} f_{*}\left(W_{n}^{\vee}\right) \rightarrow \mathbf{R}_{\mathrm{dR}}^{0} f_{*}\left(W_{n+1}^{\vee}\right) \rightarrow \ldots
$$

we simply note that the given map restricts to an isomorphism on the fibre over $s$, and is hence an isomorphism. For the second, we simply use the long exact sequence in cohomology and the inductive hypothesis for $\mathbf{R}_{\mathrm{dR}}^{1} f_{*}\left(W_{n}\right)$ and $\mathbf{R}_{\mathrm{dR}}^{1} f_{*}\left(W_{n}^{\vee}\right)$. For the third, note that it follows from the construction of $W_{n+1}$ that the exact sequence

$$
0 \rightarrow p^{*} W_{n}^{\vee} \rightarrow p^{*} W_{n+1}^{\vee} \rightarrow\left(\mathbf{R}_{\mathrm{dR}}^{1} f_{*} W_{n}^{\vee}\right)^{\vee} \rightarrow 0
$$

splits when we push out via the map $p^{*} W_{n}^{\vee} \rightarrow \mathcal{O}_{S}$. This splitting induces a map $p^{*} W_{n+1}^{\vee} \rightarrow \mathcal{O}_{S}$ such that the diagram

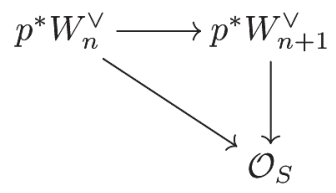

commutes. Now the fact that the diagram

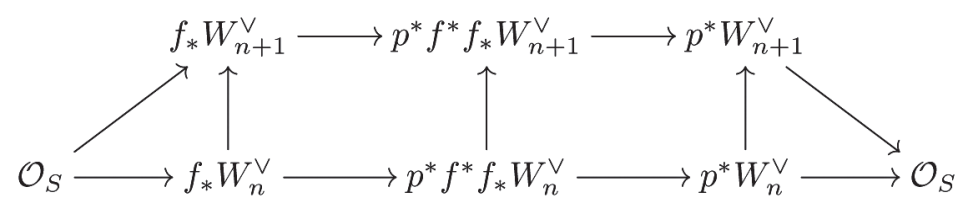

commutes implies that the composite along the top row is an isomorphism, finishing the proof.

COROLlary 1.20. Let $f: X \rightarrow S$ be a 'good' morphism over an algebraically closed field of characteristic zero, and $p$ a section of $f$. Then the natural 'base change' map $\pi_{1}^{\mathrm{dR}}\left(X_{s}, p_{s}\right) \rightarrow \pi_{1}^{\mathrm{dR}}(X / S, p)_{s}$ is an isomorphism.

REMARK 1.21. It is possible to define a relative fundamental group when $k$ is not necessarily algebraically closed (but still of characteristic 0) using identical methods. One can then show that the corresponding 'base change' question can be deduced from what we have proved in the algebraically closed case. Since this argument is rather fiddly, and not necessary in the context of this paper, we have omitted it. 


\section{Path torsors, non-abelian crystals and period maps}

If $\mathcal{T}$ is a Tannakian category over an arbitrary field $k$, and $\omega_{i}$ are fibre functors on $\mathcal{T}, i=1,2$, with values in the category of quasi-coherent sheaves on some $k$ scheme $S$, then the functor of isomorphisms $\omega_{1} \rightarrow \omega_{2}$ is representable by an affine $S$-scheme, which is a $\left(G\left(\mathcal{T}, \omega_{1}\right), G\left(\mathcal{T}, \omega_{2}\right)\right)$-bitorsor. This allows us to define path torsors under the algebraic and de Rham fundamental groups. In this section, we show how to do this in the relative case.

\section{1 - Torsors in Tannakian categories}

Let $\mathcal{C}$ be a Tannakian category over a field $k$. A Tannakian $\mathcal{C}$-category is a Tannakian category $\mathcal{D}$ together with an exact, $k$-linear tensor functor $t: \mathcal{C} \rightarrow \mathcal{D}$. We say it is neutral over $\mathcal{C}$ if there exists an exact, faithful $k$-linear tensor functor $\omega: \mathcal{D} \rightarrow \mathcal{C}$ such that $\omega \circ t \cong \mathrm{id}$. Such functors will be called fibre functors. If such a functor $\omega$ is fixed, we say $\mathcal{D}$ is neutralised. Thanks to $\$ 6.4$ of [19], we have a homomorphism

$$
t^{*}: \pi(\mathcal{D}) \rightarrow t(\pi(\mathcal{C}))
$$

of affine group schemes over $\mathcal{D}$. Hence applying $\omega$ gives us a homomorphism

$$
\omega\left(t^{*}\right): \omega(\pi(\mathcal{D})) \rightarrow \pi(\mathcal{C})
$$

of affine group schemes over $\mathcal{C}$. We define $G(\mathcal{D}, \omega):=\operatorname{ker} \omega\left(t^{*}\right)$.

For an affine group scheme $G$ over $\mathcal{C}$, let $\mathcal{O}_{G}$ be its Hopf algebra, a representation of $G$ is then defined to be an $\mathcal{O}_{G}$-comodule. That is an object $V \in \mathcal{C}$ together with a map $\delta: V \rightarrow \mathcal{O}_{G} \otimes V$ satisfying the usual axioms.

Definition 2.1. A torsor under $G$ is a non-empty affine scheme $\operatorname{Sp}\left(\mathcal{O}_{\mathrm{P}}\right)$ over $\mathcal{C}$, together with a $\mathcal{O}_{G}$-comodule structure on $\mathcal{O}_{P}$, such that the induced map $\mathcal{O}_{P} \otimes \mathcal{O}_{P} \rightarrow \mathcal{O}_{P} \otimes \mathcal{O}_{G}$ is an isomorphism.

EXAmple 2.2. Suppose that $\mathcal{C}=\operatorname{Rep}_{k}(H)$, for some affine group scheme $H$ over $k$. Then an affine group scheme $G$ over $\mathcal{C}$ 'is' just an affine group scheme $G_{0}$ over $k$ together with an action of $H$. A representation of $G$ 'is' then just an $H$ equivariant representation of $G_{0}$, or in other words, a representation of the semidirect product $G_{0} \times H$.

Representations have another interpretation. Suppose that $V$ is an $\mathcal{O}_{G}$-comodule, and let $R$ be a $\mathcal{C}$-algebra. A point $g \in G(R)$ is then a morphism $\mathcal{O}_{G} \rightarrow R$ of $\mathcal{C}$ algebras, and hence for any such $g$ we get a morphism

$$
V \rightarrow V \otimes R
$$


which extends linearly to a morphism

$$
V \otimes R \rightarrow V \otimes R
$$

This is an isomorphism, with inverse given by the map induced by $g^{-1}$. Hence we get an $R$-linear action of $G(R)$ on $V \otimes R$, for all $\mathcal{C}$-algebras $R$. The same proof as in the absolute case (Proposition 2.2 of [31]) shows that a representation of $G$ (defined in terms of comodules) is equivalent to an $R$-linear action of $G(R)$ on $V \otimes R$, for all $R$.

For $G$ an affine group scheme over $\mathcal{C}$, let $\operatorname{Rep}_{\mathcal{C}}(G)$ denote its category of representations, which is a Tannakian category over $k$. There are canonical functors

$$
\mathcal{C} \underset{\omega}{\stackrel{t}{\rightleftarrows}} \operatorname{Rep}_{\mathcal{C}}(G)
$$

given by 'trivial representation' and 'forget the representation'. This makes $\operatorname{Rep}_{\mathcal{C}}(G)$ neutral over $\mathcal{C}$. There is a natural homomorphism $G \rightarrow \omega\left(\pi\left(\operatorname{Rep}_{\mathcal{C}}(G)\right)\right)$ which comes from the fact that by definition, $G$ acts on $\omega(V)$ for all $V \in \operatorname{Rep}_{\mathcal{C}}(G)$. Since this action is trivial on everything of the form $t(W), W \in \mathcal{C}$, again by definition, this homomorphism factors to give a homomorphism

$$
G \rightarrow G\left(\operatorname{Rep}_{\mathcal{C}}(G), \omega\right)
$$

Conversely, if $\mathcal{D}$ is neutral over $\mathcal{C}$, with fibre functor $\omega$, then the action of $\omega(\pi(\mathcal{D}))$ on $\omega(V)$, for all $V \in \mathcal{D}$, induces an action of $G(\mathcal{D}, \omega)$ on $\omega(V)$, and hence a functor

$$
\mathcal{D} \rightarrow \operatorname{Rep}_{\mathcal{C}}(G(\mathcal{D}, \omega)) .
$$

Proposition 2.3. In the above situation, the homomorphism

$$
G \rightarrow G\left(\operatorname{Rep}_{\mathcal{C}}(G), \omega\right)
$$

is an isomorphism, and the functor

$$
\mathcal{D} \rightarrow \operatorname{Rep}_{\mathcal{C}}(G(\mathcal{D}, \omega))
$$

is an equivalence of categories.

Proof. Suppose first that $\mathcal{C} \cong \operatorname{Rep}_{k}(H)$ is neutral. In the first case, we can identify $G$ with an affine group scheme $G_{0}$ over $k$ together with an action of $H$, and the category $\operatorname{Rep}_{\mathcal{C}}(G)$ with the category of representations of the semi-direct product $G_{0} \times H$. The functor $\omega: \operatorname{Rep}_{\mathcal{C}}(G) \rightarrow \mathcal{C}$ can be identified with the forgetful functor from $G_{0} \times H$-representations to $H$-representations, and the morphism

$$
\omega\left(\pi\left(\operatorname{Rep}_{\mathcal{C}}(G)\right)\right) \rightarrow \pi(\mathcal{C})
$$

with the natural map

$$
G_{0} \times H \rightarrow H
$$

of affine group schemes with $H$-action. Thus the kernel of this map is identified with 
$G_{0}$ together with its given $H$-action. In other words,

$$
G \rightarrow G\left(\operatorname{Rep}_{\mathcal{C}}(G), \omega\right)
$$

is an isomorphism.

In the second case, $\mathcal{D}$ is also neutral, and corresponds to representations of some affine group scheme $G$. The functors $t, \omega$ give a surjection $G \rightarrow H$ and a splitting $H \rightarrow G$ which induces an action of $H$ on $G_{0}:=\operatorname{ker}(G \rightarrow H)$ such that $G \cong G_{0} \rtimes H$. Then $G(D, \omega)$ is identified with $G_{0}$ together with its $H$-action, and $\mathcal{D} \rightarrow \operatorname{Rep}_{\mathcal{C}}(G(\mathcal{D}, \omega))$ with the natural functor from $G=G_{0} \rtimes H$-representations to $H$-equivariant $G_{0}$-representations. It is thus an equivalence.

If $\mathcal{C}$ is not neutral, then we can choose a fibre functor with values in some $k$ scheme $S$, apply Théorème 1.12 of [20] and replace the affine group scheme $H$ by a certain groupoid acting on a $S$ (for more details see §3.3). The argument is then formally identical.

REMARK 2.4. Our definition of the fundamental group $\pi_{1}^{\mathrm{dR}}(X / S, p)$ is then just $G\left(\mathcal{N}_{f} \mathrm{IC}(X), p^{*}\right)$, as an affine group scheme over $\operatorname{IC}(S)$.

In order to define torsors of isomorphisms in the relative setting, we must first recall Deligne's construction in the absolute case, which uses the notion of a coend. So suppose that we have categories $\mathcal{X}$ and $\mathcal{S}$, and a functor $F: \mathcal{X} \times \mathcal{X}^{\mathrm{op}} \rightarrow \mathcal{S}$. The coend of $F$ is the universal pair $(\zeta, s)$ where $s$ is an object of $\mathcal{S}$ and $\zeta: F \rightarrow s$ is a binatural transformation. Here $s$ is the constant functor at $s \in \mathrm{Ob}(\mathcal{S})$, and by binatural we mean that it is natural in both variables. If such an object exists, we will denote it by

$$
\int^{\mathcal{X}} F(x, x)
$$

If $\mathcal{S}$ is cocomplete then the coend always exists and is given concretely by the formula (see Chapter IX, Section 6 of [30])

$$
\int^{\mathcal{X}} F(x, x)=\operatorname{colim}\left(\coprod_{f: x \rightarrow y \in \operatorname{Mor}(\mathcal{X})} F(x, y) \rightrightarrows \coprod_{x \in \mathrm{Ob}(\mathcal{X})} F(x, x)\right)
$$

Suppose that $\mathcal{C}$ is a Tannakian category, and let $\omega_{1}, \omega_{2}: \mathcal{C} \rightarrow \mathrm{QCoh}(S)$ be two fibre functors on $\mathcal{C}$. In [20], Deligne defines

$$
L_{S}\left(\omega_{1}, \omega_{2}\right)=\int^{\mathcal{C}} \omega_{1}(V) \otimes \omega_{2}(V)^{\vee}
$$

to be the coend of the bifunctor

$$
\omega_{1} \otimes \omega_{2}^{\vee}: \mathcal{C} \times \mathcal{C}^{\mathrm{op}} \rightarrow \mathrm{QCoh}(S),
$$


and in $\S 6$ of loc. cit., uses the tensor structure of $\mathcal{C}$ to define a multiplication on $L_{S}\left(\omega_{1}, \omega_{2}\right)$ which makes it into a quasi-coherent $\mathcal{O}_{S}$-algebra. He then proves that $\operatorname{Spec}\left(L_{S}\left(\omega_{1}, \omega_{2}\right)\right)$ represents the functor of isomorphisms from $\omega_{1}$ to $\omega_{2}$.

Now let $\mathcal{C}$ be a Tannakian category, let $\mathcal{D}$ be neutral over $\mathcal{C}$, and suppose that $\omega_{1}, \omega_{2}: \mathcal{D} \rightarrow \mathcal{C}$ are two fibre functors from $\mathcal{D}$ to $\mathcal{C}$. Define the coend

$$
L_{\mathcal{C}}\left(\omega_{1}, \omega_{2}\right):=\int^{\mathcal{D}} \omega_{1}(V) \otimes \omega_{2}(V)^{\vee} \in \operatorname{Ind}(\mathcal{C}) .
$$

If $\eta: \mathcal{C} \rightarrow \mathrm{QCoh}(S)$ is a fibre functor, then $\eta$ commutes with colimits, and hence $\eta\left(L_{\mathcal{C}}\left(\omega_{1}, \omega_{2}\right)\right)=L_{S}\left(\eta \omega_{1}, \eta \omega_{2}\right)$ : this is a quasi-coherent $\mathcal{O}_{S}$-algebra, functorial in $\eta$. Since algebraic structures in Tannakian categories, such as commutative algebras, Hopf algebras, and so on, can be constructed 'functorially in fibre functors', (see for example $\$ 5.11$ of [19]), it follows that there is a unique way of defining a $\mathcal{C}$-algebra structure on $L_{\mathcal{C}}\left(\omega_{1}, \omega_{2}\right)$ lifting the $\mathcal{O}_{S}$-algebra structure on each $\eta\left(L_{\mathcal{C}}\left(\omega_{1}, \omega_{2}\right)\right)$. Moreover, since $\eta\left(\operatorname{Sp}\left(L_{\mathcal{C}}\left(\omega_{1}, \omega_{2}\right)\right)\right)$ is a $\left(\eta \omega_{1}(\pi(\mathcal{D})), \eta \omega_{2}(\pi(\mathcal{D}))\right)$-bitorsor, functorially in $\eta$, the affine scheme

$$
P_{\mathcal{C}}\left(\omega_{1}, \omega_{2}\right):=\operatorname{Sp}\left(L_{\mathcal{C}}\left(\omega_{1}, \omega_{2}\right)\right)
$$

is a $\left(\omega_{1}(\pi(\mathcal{D})), \omega_{2}(\pi(\mathcal{D}))\right)$-bitorsor over $\mathcal{C}$.

What we actually want, however, is a $\left(G_{\mathcal{C}}\left(\mathcal{D}, \omega_{2}\right), G_{\mathcal{C}}\left(\mathcal{D}, \omega_{2}\right)\right)$-bitorsor. We get this as follows. Suppose that $V \in \mathcal{D}$, then by the definition of $L_{\mathcal{C}}\left(\omega_{1}, \omega_{2}\right)$ we get a morphism

$$
\omega_{1}(V) \otimes \omega_{2}(V)^{\vee} \rightarrow L_{\mathcal{C}}\left(\omega_{1}, \omega_{2}\right)
$$

which corresponds to a morphism

$$
\omega_{1}(V) \rightarrow \omega_{2}(V) \otimes L_{\mathcal{C}}\left(\omega_{1}, \omega_{2}\right) .
$$

Thus a morphism $L_{\mathcal{C}}\left(\omega_{1}, \omega_{2}\right) \rightarrow R$ for some $\mathcal{C}$-algebra $R$ induces an $R$-linear morphism

$$
\omega_{1}(V) \otimes R \rightarrow \omega_{2}(V) \otimes R
$$

which is in fact an isomorphism, since it is so after applying any fibre functor.

Definition 2.5. Define $P_{\text {triv }}\left(\omega_{1}, \omega_{2}\right)$ to be the sub-functor of $P_{\mathcal{C}}\left(\omega_{1}, \omega_{2}\right)$ which takes $R$ to the set of all morphisms $L_{\mathcal{C}}\left(\omega_{1}, \omega_{2}\right) \rightarrow R$ such that for every $V$ in the essential image of $t: \mathcal{C} \rightarrow \mathcal{D}$, the induced automorphism of $R \otimes \omega_{1}(V)=R \otimes \omega_{2}(V)$ is the identity.

Proposition 2.6. The functor $P_{\text {triv }}\left(\omega_{1}, \omega_{2}\right)$ is representable by an affine scheme over $\mathcal{C}$, and is a $\left(G_{\mathcal{C}}\left(\mathcal{D}, \omega_{1}\right), G_{\mathcal{C}}\left(\mathcal{D}, \omega_{2}\right)\right)$-bitorsor in the category of affine schemes over $\mathcal{C}$. 
Proof. First note that if $V \in \operatorname{Ob}(\mathcal{D})$, then $\omega_{i}(\pi(\mathcal{D}))$ acts on $\omega_{i}(V)$, and $G\left(\mathcal{D}, \omega_{i}\right)$ is the largest subgroup of $\omega_{i}(\pi(\mathcal{D}))$ whose action on $\omega_{i}(V)$ is trivial for all $V$ in the essential image of $t$.

Now, if $p \in P_{\text {triv }}\left(\omega_{1}, \omega_{2}\right)(R)$ and $g \in G_{\mathcal{C}}\left(\mathcal{D}, \omega_{1}\right)(R)$ then $g p \in P_{\mathcal{C}}\left(\omega_{1}, \omega_{2}\right)(R)$ acts trivially on everything of the form $t(W)$, and hence lies in $P_{\text {triv }}\left(\omega_{1}, \omega_{2}\right)(R)$. Hence $G\left(\mathcal{D}, \omega_{1}\right)$ acts on $P_{\text {triv }}\left(\omega_{1}, \omega_{2}\right)$. For $p, p^{\prime} \in P_{\text {triv }}\left(\omega_{1}, \omega_{2}\right)(R), p^{-1} p^{\prime}$ is an automorphism of $\omega_{1}(V) \otimes R$ which is trivial for all $V$ in the essential image of $t$. Hence it must be an element of $G\left(\mathcal{D}, \omega_{1}\right)(R) \subset \omega\left(\pi_{1}(\mathcal{D})\right)(R)$. The same arguments work for $G_{\mathcal{C}}\left(\mathcal{D}, \omega_{2}\right)$.

Thus $P_{\text {triv }}\left(\omega_{1}, \omega_{2}\right)$ is a bi-pseudo-torsor, and to complete the proof, we must show that $P_{\text {triv }}\left(\omega_{1}, \omega_{2}\right)$ is represented by a non-empty affine scheme over $\mathcal{C}$. Now, consider the ind-object of $\mathcal{C}$

$$
L_{\mathcal{C}}(\mathrm{id}, \mathrm{id})=\operatorname{colim}\left(\coprod_{f: V \rightarrow W \in \operatorname{Mor}(\mathcal{C})} V \otimes W^{\vee} \rightrightarrows \coprod_{V \in \operatorname{Ob}(\mathcal{C})} V \otimes V^{\vee}\right) .
$$

For every fibre functor $\eta: \mathcal{C} \rightarrow \mathrm{QCoh}(S)$, there is a natural Hopf algebra structure on $\eta\left(L_{\mathcal{C}}(\mathrm{id}, \mathrm{id})\right)=L_{S}(\eta, \eta)$, and the Spec of this Hopf algebra is the affine group scheme of tensor automorphisms of $\eta$. Thus as before, $L_{\mathcal{C}}(\mathrm{id}$, id) has a Hopf algebra structure, and its formal Spec satisfies the defining property of the fundamental groupoid $\pi(\mathcal{C})$ of $\mathcal{C}$. Hence one can construct a morphism of affine $\mathcal{C}$-schemes

$$
P_{\mathcal{C}}\left(\omega_{1}, \omega_{2}\right) \rightarrow \pi(\mathcal{C})
$$

which is the formal Spec of the obvious morphism $L_{\mathcal{C}}(\mathrm{id}, \mathrm{id}) \rightarrow L_{\mathcal{C}}\left(\omega_{1}, \omega_{2}\right)$. Then $P_{\text {triv }}\left(\omega_{1}, \omega_{2}\right)$ is the fibre of $P_{\mathcal{C}}\left(\omega_{1}, \omega_{2}\right) \rightarrow \pi(\mathcal{C})$ over the identity section $\operatorname{Sp}(1) \rightarrow \pi(\mathcal{C})$. Hence it is the formal Spec of the algebra $L_{\text {triv }}\left(\omega_{1}, \omega_{2}\right)$ defined by the push-out diagram

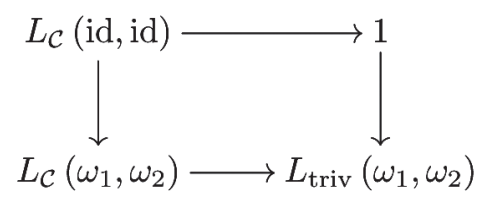

and is thus representable by an affine $\mathcal{C}$-scheme.

To prove that $P_{\text {triv }}\left(\omega_{1}, \omega_{2}\right) \neq \emptyset$, it suffices to show that $\eta\left(P_{\text {triv }}\left(\omega_{1}, \omega_{2}\right)\right) \neq \emptyset$ for any fibre functor $\eta: \mathcal{C} \rightarrow \mathrm{QCoh}(S)$. For any $f: T \rightarrow S, \eta\left(P_{\text {triv }}\left(\omega_{1}, \omega_{2}\right)\right)(T)$ is the subset of $\operatorname{Isom}^{\otimes}\left(f^{*} \circ \eta \omega_{1}, f^{*} \circ \eta \omega_{2}\right)$ which maps to the identity under the natural map

(71) $\quad r: \operatorname{Isom}^{\otimes}\left(f^{*} \circ \eta \omega_{1}, f^{*} \circ \eta \omega_{2}\right) \rightarrow \operatorname{Isom}^{\otimes}\left(f^{*} \circ \eta \omega_{1} t, f^{*} \circ \eta \omega_{2} t\right)=\operatorname{Aut}^{\otimes}\left(f^{*} \circ \eta\right)$.

Since $\eta \omega_{1}$ and $\eta \omega_{2}$ are fibre functors on $\mathcal{D}$, according to 1.13 of [20] the affine scheme $\underline{\operatorname{Isom}}^{\otimes}\left(\eta \omega_{1}, \eta \omega_{2}\right)$ of tensor isomorphisms from $\eta \omega_{1}$ to $\eta \omega_{2}$ is faithfully flat 
over $S$, and in particular is non-empty. Hence there is certainly some $S$-scheme $f: T \rightarrow S$ such that

$$
\operatorname{Isom}^{\otimes}\left(f^{*} \circ \eta \omega_{1}, f^{*} \circ \eta \omega_{2}\right)
$$

is non-empty. Pick such a $T$, and pick some $p \in \operatorname{Isom}^{\otimes}\left(f^{*} \circ \eta \omega_{1}, f^{*} \circ \eta \omega_{2}\right)$. Since the morphism $\omega_{1}(\pi(\mathcal{D})) \rightarrow \pi(\mathcal{C})$ admits a section, the induced homomorphism

$$
\operatorname{Aut}^{\otimes}\left(f^{*} \circ \eta \omega_{1}\right) \rightarrow \operatorname{Aut}^{\otimes}\left(f^{*} \circ \eta\right)
$$

is surjective, and hence there exists some $g \in \operatorname{Aut}^{\otimes}\left(f^{*} \circ \eta \omega_{1}\right)$ mapping to $r(p) \in$ $\operatorname{Aut}^{\otimes}\left(f^{*} \circ \eta\right)$. Then $p^{\prime}:=g^{-1} p$ is an element of the set $\operatorname{Isom}^{\otimes}\left(f^{*} \circ \eta \omega_{1}, f^{*} \circ \eta \omega_{2}\right)$ and $r\left(p^{\prime}\right)=\mathrm{id}$, thus $\eta\left(P_{\text {triv }}\left(\omega_{1}, \omega_{2}\right)\right)(T) \neq \emptyset$.

REMARK 2.7. We can rephrase this as follows. Consider the functors of $\mathcal{C}$ algebras

$$
\begin{aligned}
\underline{\mathrm{Isom}}^{\otimes}\left(\omega_{1}, \omega_{2}\right): \mathcal{C}-\mathrm{alg} & \rightarrow(\text { Set }) \\
R & \mapsto \operatorname{Isom}^{\otimes}\left(\omega_{1}(-) \otimes R, \omega_{2}(-) \otimes R\right) ; \\
\underline{\operatorname{Aut}}^{\otimes}(\mathrm{id}): \mathcal{C}-\mathrm{alg} & \rightarrow(\text { Set }) \\
R & \mapsto \operatorname{Aut}^{\otimes}((-) \otimes R) ;
\end{aligned}
$$

as well as the sub-functor $\underline{\operatorname{Isom}}_{\mathcal{C}}^{\otimes}\left(\omega_{1}, \omega_{2}\right)$, the 'functor of $\mathcal{C}$-isomorphisms $\omega_{1} \rightarrow \omega_{2}$ ', defined to be the fibre over the identity of the natural morphism

$$
\underline{\mathrm{Isom}}^{\otimes}\left(\omega_{1}, \omega_{2}\right) \rightarrow{\underline{\text { Aut }^{\otimes}}}^{(\mathrm{id}) .}
$$

Then the functor $\underline{\operatorname{Isom}}_{\mathcal{C}}^{\otimes}\left(\omega_{1}, \omega_{2}\right)$ is representable by the affine scheme $P_{\text {triv }}\left(\omega_{1}, \omega_{2}\right)$ over $\mathcal{C}$, which is a $\left(G_{\mathcal{C}}\left(\mathcal{D}, \omega_{1}\right), G_{\mathcal{C}}\left(\mathcal{D}, \omega_{2}\right)\right)$ bitorsor.

\section{2 - Path torsors under relative fundamental groups}

Let $k$ be an algebraically closed field of characteristic zero, $S$ a connected, smooth variety over $k$ and $f: X \rightarrow S$ a 'good' morphism. Let $p, x$ be sections of $f$. We can apply the above methods to obtain an affine scheme over $\operatorname{IC}(S)$, the torsor of paths from $x$ to $p$, which can be considered as an affine scheme $P(x)=$ $\pi_{1}^{\mathrm{dR}}(X / S, x, p)$ over $S$, together with an integrable connection on $\mathcal{O}_{P(x)}$ (as a quasicoherent $\mathcal{O}_{S}$-algebra). This is naturally a left torsor under $\pi_{1}^{\mathrm{dR}}(X / S, x)$ and a right torsor under $\pi_{1}^{\mathrm{dR}}(X / S, p)=: G$. Moreover, the action map $P(x) \times G \rightarrow P(x)$ is compatible with the connections, in the sense that the associated comodule structure

$$
\mathcal{O}_{P(x)} \rightarrow \mathcal{O}_{P(x)} \otimes_{\mathcal{O}_{S}} \mathcal{O}_{G}
$$

is horizontal, the RHS being given the tensor product connection. If $G_{n}$ is the 
quotient of $G$ by the $n$th term in its lower central series, we will denote the push-out torsor $P(x) \times{ }^{G} G_{n}$ by $P(x)_{n}$. As before, the action map $P(x)_{n} \times G_{n} \rightarrow P(x)_{n}$ is compatible with the connections.

Definition 2.8. A $\nabla$-torsor under $G_{n}$ is a $G_{n}$-torsor $P$ over $S$ in the usual sense, together with a regular integrable connection on $\mathcal{O}_{P}$, such that the action map

$$
\mathcal{O}_{P} \rightarrow \mathcal{O}_{P} \otimes \mathcal{O}_{G_{n}}
$$

is horizontal. The set of isomorphism classes of $\nabla$-torsors is denoted $H_{\nabla}^{1}\left(S, G_{n}\right)$.

Thus we have 'period maps'

$$
X(S) \rightarrow H_{\nabla}^{1}\left(S, G_{n}\right)
$$

which takes $x \in X(S)$ to the path torsor $P(x)_{n}$.

REMARK 2.9. (1) This is not a good period map to study. For instance, if $k=\mathbb{C}$, then the relative fundamental group is not just an affine group scheme with connection. There are reasons to expect that one can put a 'non-abelian' variation of Hodge structure on this fundamental group. Similar considerations will apply to the path torsors, and the period maps should take these variations of Hodge structures into account.

(2) We can use the pro-nilpotent Lie algebra of $\pi_{1}^{\mathrm{dR}}(X / S, p)$ and the CampbellHausdorff law to view $\pi_{1}^{\mathrm{dR}}(X / S, p)$ as a non-abelian sheaf of groups on the infinitesimal site of $S / k$. We can use this interpretation to give an alternative definition of the cohomology set $H_{\nabla}^{1}\left(S, G_{n}\right)$.

(3) A natural question to ask is whether or not, as in the situation studied by Kim, the targets for the period maps have the structure of algebraic varieties. Since we are more interested in the positive characteristic case, we will not pursue this question here.

\section{Crystalline fundamental groups of smooth families in char $p$}

Our goal in this section is to define the fundamental group of a smooth family $f: X \rightarrow S$ of varieties over a finite field. Many of our arguments are essentially the same as those we gave in Section 1.

We will assume that the reader is familiar with the theory of rigid cohomology and overconvergent $(F$-)isocrystals, a good reference is [2]. Assume that $k$ is a finite field, of order $q=p^{a}$ and characteristic $p>0$. Frobenius will always refer to linear Frobenius. If $U / K$ is a variety, the category of overconvergent $(F$-)isocrystals on $U / K$ is denoted $(F-) \operatorname{Isoc}{ }^{\dagger}(U / K)$. These are Tannakian categories over $K$.

We define $\mathcal{N} \operatorname{Isoc}^{\dagger}(U / K)$ to be the full subcategory of $\operatorname{Isoc}^{\dagger}(U / K)$ on objects admitting a filtration whose graded pieces are constant. Chiarellotto and Le Stum 
in [16] define the rigid fundamental group $\pi_{1}^{\text {rig }}(U, x)$ of $U$ at a $k$-rational point $x$ to be the Tannaka dual of $\mathcal{N} \operatorname{Isoc}^{\dagger}(U / K)$ with respect to the fibre functor $x^{*}$. This is a pro-unipotent group scheme over $K$.

Now suppose that $g: X \rightarrow S$ is a 'good', proper morphism over $k$, and let $p: S \rightarrow X$ be a section.

Definition 3.1. We say that $E \in F$-Isoc ${ }^{\dagger}(X / K)$ is relatively unipotent if there is a filtration of $E$, whose graded pieces are all in the essential image of $g^{*}: F$-Isoc ${ }^{\dagger}(S / K) \rightarrow F$-Isoc ${ }^{\dagger}(X / K)$. The full subcategory of relatively unipotent overconvergent $F$-isocrystals is denoted $\mathcal{N}_{g} F$-Isoc ${ }^{\dagger}(X / K)$.

The pair of functors

$$
\mathcal{N}_{g} F-\operatorname{Isoc}^{\dagger}(X / K) \underset{g^{*}}{\stackrel{p^{*}}{\rightleftarrows}} F-\operatorname{Isoc}^{\dagger}(S / K)
$$

makes $\mathcal{N}_{g} F$-Isoc ${ }^{\dagger}(X / K)$ neutral over $F$-Isoc ${ }^{\dagger}(S / K)$ in the sense of $\S 2.1$. Hence we get an affine group scheme $G\left(\mathcal{N}_{g} F-\operatorname{Isoc}^{\dagger}(X / K), p^{*}\right)$ in $F-\operatorname{Iscc}^{\dagger}(S / K)$.

Definition 3.2. We define the relative fundamental group to be the affine group scheme $G\left(\mathcal{N}_{g} F-\operatorname{Isoc}^{\dagger}(X / K), p^{*}\right)$ in $F-\operatorname{Isoc}^{\dagger}(S / K)$.

For $s \in S$ a closed point, let $i_{s}: X_{s} \rightarrow X$ denote the inclusion of the fibre over $s$ and let $g_{s}: X_{s} \rightarrow \operatorname{Spec} k(s)$ denote the structure morphism. Let $K(s)$ denote the unique unramified extension of $K$ with residue field $k(s)$. Let $\mathcal{V}(s)$ denote the ring of integers of $K(s)$. In keeping with notation of previous sections, let $\pi_{1}^{\text {rig }}(X / S, p)_{s}$ denote the affine group scheme $s^{*}\left(\pi_{1}^{\mathrm{rig}}(X / S, p)\right)$ over $K(s)$. The pull-back functor

$$
i_{s}^{*}: \mathcal{N}_{g} F-\operatorname{Isoc}^{\dagger}(X / K) \rightarrow \mathcal{N} \operatorname{Isoc}^{\dagger}\left(X_{s} / K(s)\right)
$$

induces a homomorphism

$$
\phi: \pi_{1}^{\mathrm{rig}}\left(X_{s}, p_{s}\right) \rightarrow \pi_{1}^{\mathrm{rig}}(X / S, p)_{s}
$$

of affine group schemes over $K$. We would like to show again that when $S$ is an affine curve, this is an isomorphism. The question is whether or not the sequence of affine group schemes corresponding to the sequence of neutral Tannakian categories

$$
\mathcal{N} \operatorname{Isoc}\left(X_{s} / K(s)\right) \leftarrow \mathcal{N}_{g} F-\operatorname{Isoc}^{\dagger}(X / K) \otimes_{K} K(s) \leftarrow F-\operatorname{Isoc}^{\dagger}(S / K) \otimes_{K} K(s)
$$

is exact. Thus, as before, this boils down to the following three questions.

(1) If $E \in \mathcal{N}_{g} F$-Isoc ${ }^{\dagger}(X / K) \otimes_{K} K(s)$ is such that $i_{s}^{*} E$ is constant, is $E$ of the form $g^{*} F$ for some $F \in F$-Isoc ${ }^{\dagger}(S / K) \otimes_{K} K(s)$ ?

(2) If $E \in \mathcal{N}_{g} F$-Isoc ${ }^{\dagger}(X / K) \otimes_{K} K(s)$, and $F_{0} \subset i_{s}^{*} E$ denotes the largest constant subobject, then does there exist $E_{0} \subset E$ such that $F_{0}=i_{s}^{*} E_{0}$ ?

(3) Given $E \in \operatorname{Isoc}^{\dagger}\left(X_{s} / K(s)\right.$ ), does there exist $F \in \mathcal{N}_{g} F$-Isoc ${ }^{\dagger}(X / K) \otimes_{K} K(s)$ such that $E$ is a quotient of $i_{s}^{*} F$ ? 
REMARK 3.3. Actually, in order to apply these criteria, we need to know that the kernel of the homomorphism of group schemes corresponding to

$$
\mathcal{N}_{g} F-\operatorname{Isoc}^{\dagger}(X / K) \otimes_{K} K(s) \leftarrow F-\operatorname{Isoc}^{\dagger}(S / K) \otimes_{K} K(s)
$$

is pro-unipotent, or using Lemma 1.3, Part I of [35], that every object $E$ of the category $\mathcal{N}_{g} F$-Isoc ${ }^{\dagger}(X / K) \otimes_{K} K(s)$ has a non-zero subobject of the form $f^{*} F$ for some $F \in F$-Isoc ${ }^{\dagger}(S / K) \otimes_{K} K(s)$. Let $E_{0}$ denote the largest relatively constant subobject of $E$, considered in the category $\mathcal{N}_{g} F$-Isoc ${ }^{\dagger}(X / K)$. Then functoriality of $E_{0}$ implies that it is invariant under endomorphisms of $E$, and hence a $K(s)$-module structure $K(s) \rightarrow \operatorname{End}_{\mathcal{N}_{g} F \text {-Isoc }(X / K)}(E)$ will induce one on $E_{0}$. Hence we must show that for $F \in F$-Isoc ${ }^{\dagger}(S / K)$, every $K(s)$-module structure on $g^{*} F$ comes from one on $F$, and it certainly suffices to show that $\operatorname{End}\left(f^{*} F\right) \cong \operatorname{End}(F)$. Using Tannakian duality we can translate this into a problem about representations of groupoids we have a surjective morphism $G \rightarrow H$ of $K$-groupoids and a representation $V$ of $H$, and we want to show that $\operatorname{End}_{H}(V) \cong \operatorname{End}_{G}\left(\left.V\right|_{G}\right)$. This is clear.

\section{1 - Base change}

Hypotheses and notations will be as in the previous section, and will make the following additional technical hypothesis.

Hypothesis 3.4. The morphism $g: X \rightarrow S$ is realisable, in the sense that there exists a commutative diagram

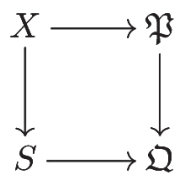

where $\mathfrak{P}, \subseteq$ are smooth and proper formal $\mathcal{V}$-schemes, both horizontal arrows are locally closed immersions and $\mathfrak{P} \rightarrow \mathfrak{Q}$ is smooth around $X$.

REMARK 3.5. (1) We should eventually be able to remove this technical hypothesis, using methods of 'recollement', but we do not worry about this for now.

(2) One non-trivial example of such a $g$ is given by a model for a smooth, proper, geometrically connected curve $C$ over a function field $K$ over a finite field. In this situation $S^{\prime}$ is the unique smooth, proper model for $K, X^{\prime}$ is a regular, flat, proper $S^{\prime}$-scheme, whose generic fibre is $C, S \subset S^{\prime}$ is an affine open subset of $S^{\prime}$ over which $g$ is smooth, and $X$ is the pre-image of $S$. Since $X^{\prime}$ is a regular, proper surface over a finite field, it is smooth, hence projective, and the above hypotheses really are satisfied.

In this section we will prove the following two theorems. 
Theorem 3.6. (1) Let $E \in \mathcal{N}_{g} F-\operatorname{Isoc}^{\dagger}(X / K) \otimes_{K} K(s)$ and suppose that $i_{s}^{*} E$ is a constant isocrystal. Then there exists $E^{\prime} \in F-\operatorname{Isoc}^{\dagger}(S / K) \otimes_{K} K(s)$ such that $E \cong g^{*} E^{\prime}$.

(2) Let $E \in \mathcal{N}_{g} F$-Isoc $\operatorname{Is}^{\dagger}(X / K) \otimes_{K} K(s)$, and let $F_{0} \subset i_{s}^{*} E$ denote the largest constant subobject. Then there exists $E_{0} \subset E$ such that $F_{0}=i_{s}^{*} E_{0}$.

THEOREM 3.7. Let $E \in \mathcal{N} \operatorname{Isoc}^{\dagger}\left(X_{s} / K(s)\right)$. Then there exists some object $E^{\prime} \in \mathcal{N}_{g} F-\operatorname{Isoc}^{\dagger}(X / K) \otimes_{K} K(s)$ such that $E$ is a quotient of $i_{s}^{*} E^{\prime}$.

REMARK 3.8. The reason we have used categories of overconvergent $F$-isocrystals rather than overconvergent isocrystals without Frobenius is that the theory of 'six operations' has only fully been developed for overconvergent $F$-isocrystals. If six operations were to be resolved for overconvergent isocrystals in general, then we would be able to deduce results for smooth fibrations over any perfect field of positive characteristic, not just over finite fields where we can linearise Frobenius.

The method of proof will be entirely analogous to the proof in characteristic 0 , replacing the algebraic $\mathcal{D}$-modules used there by their arithmetic counterparts, the theory of which was developed by Berthelot and Caro. It would be far too much of a detour to describe this theory in any depth, so instead we will just recall the notations and results needed, referring the reader to the series of articles [5], [3], [4] and [10], [9], [6], [12], [8], [7] for details.

We let $F$ - $D_{\text {surhol }}^{b}\left(\mathcal{D}_{X / K}\right)$ (resp. $\left.F-D_{\text {surhol }}^{b}\left(\mathcal{D}_{S / K}\right)\right)$ denote the category of overholonomic $F$-D-modules on $X$ (resp. $S$ ) as defined in Section 3 of [9]. There is a functor

$$
\operatorname{sp}_{X,+}: F-\operatorname{Isoc}^{\dagger}(X / K) \rightarrow F-D_{\text {surhol }}^{b}\left(\mathcal{D}_{X / K}\right)
$$

which is an equivalence onto the full subcategory $F$-Isoc ${ }^{\dagger \dagger}(X / K)$ of overcoherent $F$ isocrystals (Theorem 2.3.16 of [13] and Théorème 2.3.1 of [8]) and compatible with the natural tensor products on both sides (Proposition 4.8 of [10]). The same also holds for $S$. Let

$$
\begin{gathered}
g_{+}: F-D_{\text {surhol }}^{b}\left(\mathcal{D}_{X / K}\right) \rightarrow F-D_{\text {surhol }}^{b}\left(\mathcal{D}_{S / K}\right) \\
g^{+}: F-D_{\text {surhol }}^{b}\left(\mathcal{D}_{S / K}\right) \rightarrow F-D_{\text {surhol }}^{b}\left(\mathcal{D}_{X / K}\right)
\end{gathered}
$$

be the adjoint functors defined in Section 3 of [9]. By Théorème 4.2.12 of [12], for any $E \in F$-Isoc ${ }^{\dagger}(X / K)$, and any $i \in Z, \mathcal{H}^{i}\left(g_{+} \operatorname{sp}_{X,+}(E)\right) \in F-\operatorname{Isoc}^{\dagger \dagger}(S / K)$ and hence we can define

$$
g_{*}:=\operatorname{sp}_{S,+}^{-1} \mathcal{H}^{-d}\left(g_{+} \operatorname{sp}_{X,+}(-)\right)(-d): F-\operatorname{Isoc}^{\dagger}(X / K) \rightarrow F-\operatorname{Isoc}^{\dagger}(S / K)
$$

where $d$ is the relative dimension of $X / S$, and $(-d)$ denotes the Tate twist. We can 
also define the higher direct images

$$
\mathbf{R}^{i} g_{*}:=\operatorname{sp}_{S,+}^{-1} \mathcal{H}^{-d+i}\left(g_{+} \operatorname{sp}_{X,+}(-)\right)(-d): F-\operatorname{Isoc}^{\dagger}(X / K) \rightarrow F-\operatorname{Isoc}^{\dagger}(S / K) .
$$

Let $s^{!}: F-D_{\text {surhol }}^{b}\left(\mathcal{D}_{S / K}\right) \rightarrow F-D_{\text {surhol }}^{b}\left(\mathcal{D}_{\text {Spec }(k(s)) / K(s)}\right)$ denote the functor defined in Section 3 of [9].

REMARK 3.9. Although Caro's functor $s !$ lands in $F$ - $D_{\text {surhol }}^{b}\left(\mathcal{D}_{\text {Spec }(k(s)) / K}\right)$ rather than $F$ - $D_{\text {surhol }}^{b}\left(\mathcal{D}_{\text {Spec }}\right)(k(s)) / K(s)$, it can be easily adapted to land in the latter category. The base change result that we use below holds in this slightly altered context.

Now, if $Y$ is any smooth, proper, realisable variety over $k$ (i.e. $Y$ can be embedded into a smooth and proper formal $\mathcal{V}$-scheme) with structure map $h: Y \rightarrow$ Spec $k$ then we can similarly define

$$
\mathbf{R}^{i} h_{*}: F-\operatorname{Isoc}^{\dagger}(Y / K) \rightarrow F-\operatorname{Isoc}^{\dagger}(k / K)
$$

where the target category is that of $K$-vector spaces equipped with an automorphism $\phi$. We also have the rigid cohomology functors

$$
H_{\text {rig }}^{i}(Y,-): F-\operatorname{Isoc}^{\dagger}(Y / K) \rightarrow F-\operatorname{Isoc}^{\dagger}(k / K)
$$

where the Frobenius action on the rigid cohomology $H_{\text {rig }}^{i}(Y, E)$ of an overconvergent $F$-isocrystal $E$ is the one induced by functoriality.

Proposition 3.10. For $Y / k$ smooth, proper and realisable there is an isomorphism of functors

$$
\mathbf{R}^{i} h_{*}(-) \cong H_{\text {rig }}^{i}(Y,-): F-\operatorname{Isoc}^{\dagger}(Y / K) \rightarrow F-\operatorname{Isoc}^{\dagger}(k / K) .
$$

Proof. Lemme 7.3.4 of [7] states that $\mathbf{R}^{i} h_{*}(-)(d) \cong H_{\text {rig }}^{i}(Y,-)$, however, it is pointed out in Remark 3.15 (iii) of [1] that this is incorrect, and needs to be altered by a Tate twist to give the claimed result.

Of course, a similar result holds over any finite extension of $k$.

Corollary 3.11. Let $s \in S$ be a closed point. There is an isomorphism of functors $s^{*} \mathbf{R}^{i} g_{*}(-) \cong H_{\text {rig }}^{i}\left(X_{s}, i_{s}^{*}(-)\right): F-\operatorname{Isoc}^{\dagger}(X / K) \rightarrow \operatorname{Vec}_{K(s)}$.

REMARK 3.12. We are deliberately ignoring Frobenius structure in the final target category of these two composite functors.

Proof. This follow from proper base change for arithmetic $\mathcal{D}$-modules (Théorème 4.4.2 of [12]), together with the identification $s^{*}=s^{!}[\operatorname{dim} S]$ for overcoherent $F$-isocrystals on $S$ (1.4.5 of [10]), and the previous proposition applied to $Y=X_{s} / k(s)$ (after forgetting the Frobenius structures). 
Proposition 3.13. For $E \in F-\operatorname{Isoc}^{\dagger}(X / K), g_{+} \operatorname{sp}_{X,+}(E)$ is concentrated in degrees $\geq-d$.

Proof. We know that $g_{+} \mathrm{Sp}_{X,+}(E)$ has overcoherent $F$-isocrystals for cohomology sheaves, and by the previous proposition, the fibre over $s$ of $\mathcal{H}^{i}\left(g_{+} \mathrm{sp}_{X,+}(E)\right)$ is zero for $i \leq-d$. Hence $\mathcal{H}^{i}\left(g_{+} \mathrm{sp}_{X,+}(E)\right)$ is zero for $i \leq-d$.

PROPOSITION 3.14. $g_{*}$ is right adjoint to $g^{*}$.

Proof. Since $g_{+}$is right adjoint to $g^{+}$, this will follow from the previous proposition provided $g^{+} \operatorname{sp}_{S,+}(-)[d](d)=\mathrm{sp}_{X,+} g^{*}(-)$. To see this, note that it follows from Proposition 6.5.2, together with 6.4.3(b) and 6.4.4(c) of [7] that $g^{\prime} \operatorname{sp}_{S,+}(-)[-d] \cong \operatorname{sp}_{X,+} g^{*}(-)$, and from 5.4 .8 of $[11]$ that $g^{!}[-d] \cong g^{+}[d](d)$ for overcoherent $F$-isocrystals on smooth $k$-varieties.

REMARK 3.15. Again, it may seem as though 5.4.8 of [11] gives $g^{!} \cong g^{+}$, however, the functors $\theta^{!}$and $\theta^{+}$defined there differ from $g^{!}$and $g^{+}$respectively by an appropriate shift and twist.

Proof of Theorem 3.6. Because $g_{*}$ and $g^{*}$ are functorial, they extend to give adjoint functors

$$
g^{*}: F-\operatorname{Isoc}^{\dagger}(S / K) \otimes_{K} K(s) \rightleftarrows \mathcal{N}_{g} F-\operatorname{Isoc}^{\dagger}(X / K) \otimes_{K} K(s): g_{*}
$$

such that (using the base change theorem as in the proof of Corollary 3.11) the counit $g^{*} g_{*} E \rightarrow E$ restricts to the counit of the adjunction

$$
-\otimes_{K(s)} \mathcal{O}_{X_{s} / K(s)}^{\dagger}: \operatorname{Vec}_{K(s)} \rightleftarrows \operatorname{Isoc}^{\dagger}\left(X_{s} / K(s)\right): H_{\text {rig }}^{0}\left(X_{s},-\right) .
$$

on the fibre over $s$. Thus exactly as in the proof of Proposition 1.12, if $i_{s}^{*} E$ is trivial, the counit $g^{*} g_{*} E \rightarrow E$ is an isomorphism on the fibre over $s$, and hence an isomorphism. Similarly, since $H_{\text {rig }}^{0}\left(X_{s}, i_{s}^{*} E\right) \cong \operatorname{Hom}_{\operatorname{Isoc}^{\dagger}(X / K(s))}\left(\mathcal{O}_{X_{s}}, i_{s}^{*} E\right)$, (see Proposition 3.16 below) exactly the same argument as in Proposition 1.13 shows that in general $H_{\text {rig }}^{0}\left(X_{s}, i_{s}^{*} E\right) \otimes_{K(s)} \mathcal{O}_{X_{s}}$ is the largest trivial sub-object of $i_{s}^{*} E$. Hence if we let $E_{0}=g^{*} g_{*} E$, then $i_{s}^{*} E_{0} \cong H_{\text {rig }}^{0}\left(X_{s}, i_{s}^{*} E\right) \otimes_{K(s)} \mathcal{O}_{X_{s}}$ is the largest trivial sub-object of $i_{s}^{*} E$, proving (2), and if $i_{s}^{*} E$ is trivial, then $E \cong E_{0}$, proving (1).

We now turn our attention to Theorem 3.7.

Proposition 3.16. Suppose that $E, E^{\prime} \in \operatorname{Isoc}^{\dagger}\left(X_{s} / K(s)\right)$. Then there are canonical isomorphisms

$$
\begin{aligned}
\operatorname{Hom}_{\operatorname{Isoc}^{\dagger}\left(X_{s} / K(s)\right)}\left(E, E^{\prime}\right) \cong H_{\text {rig }}^{0}\left(X_{s}, \mathcal{H o m}\left(E, E^{\prime}\right)\right) \\
\operatorname{Ext}_{\operatorname{Isoc}^{\dagger}\left(X_{s} / K(s)\right)}\left(E, E^{\prime}\right) \cong H_{\text {rig }}^{1}\left(X_{s}, \mathcal{H o m}\left(E, E^{\prime}\right)\right)
\end{aligned}
$$


and moreover if $E, E^{\prime}$ have Frobenius structures, this induces an isomorphism

$$
\operatorname{Hom}_{F-\operatorname{Isoc}^{\dagger}\left(X_{s} / K(s)\right)}\left(E, E^{\prime}\right) \cong H_{\text {rig }}^{0}\left(X_{s}, \mathcal{H o m}\left(E, E^{\prime}\right)\right)^{\phi=1}
$$

as well as a surjection

$$
\operatorname{Ext}_{F-\operatorname{Isoc}^{\dagger}\left(X_{s} / K(s)\right)}\left(E, E^{\prime}\right) \rightarrow H_{\text {rig }}^{1}\left(X_{s}, \mathcal{H o m}\left(E, E^{\prime}\right)\right)^{\phi=1}
$$

Proof. The first isomorphism is clear, and this immediately implies the third. The second is Proposition 1.3.1 of [17], from which the fourth is then easily deduced.

We define the $U_{n}$ inductively as follows. $U_{1}$ will just be $\mathcal{O}_{X_{s}}^{\dagger}$, and $U_{n+1}$ will be the extension of $U_{n}$ by $\mathcal{O}_{X_{s}}^{\dagger} \otimes_{K(s)} H_{\text {rig }}^{1}\left(X_{s}, U_{n}^{\vee}\right)^{\vee}$ corresponding to the identity under the isomorphisms

$$
\begin{aligned}
\operatorname{Ext}_{\text {Isoc }^{\dagger}\left(X_{s} / K(s)\right)} & \left(U_{n}, \mathcal{O}_{X_{s}}^{\dagger} \otimes_{K(s)} H_{\text {rig }}^{1}\left(X_{s}, U_{n}^{\vee}\right)^{\vee}\right) \\
& \cong H_{\text {rig }}^{1}\left(X_{s}, U_{n}^{\vee} \otimes_{K(s)} H_{\text {rig }}^{1}\left(X_{s}, U_{n}^{\vee}\right)^{\vee}\right) \\
& \cong H_{\text {rig }}^{1}\left(X_{s}, U_{n}^{\vee}\right) \otimes_{K(s)} H_{\text {rig }}^{1}\left(X_{s}, U_{n}^{\vee}\right)^{\vee} \\
& \cong \operatorname{End}_{K(s)}\left(H_{\text {rig }}^{1}\left(X_{s}, U_{n}^{\vee}\right)\right) .
\end{aligned}
$$

If we look at the long exact sequence in cohomology associated to the short exact sequence $0 \rightarrow U_{n}^{\vee} \rightarrow U_{n+1}^{\vee} \rightarrow \mathcal{O}_{X_{s}}^{\dagger} \otimes_{K(s)} H_{\text {rig }}^{1}\left(X_{s}, U_{n}^{\vee}\right) \rightarrow 0$ we get

$$
\begin{aligned}
0 \rightarrow H_{\text {rig }}^{0}\left(X_{s}, U_{n}^{\vee}\right) & \rightarrow H_{\text {rig }}^{0}\left(X_{s}, U_{n+1}^{\vee}\right) \rightarrow H_{\text {rig }}^{1}\left(X_{s}, U_{n}^{\vee}\right) \\
& \stackrel{\delta}{\rightarrow} H_{\text {rig }}^{1}\left(X_{s}, U_{n}^{\vee}\right) \rightarrow H_{\text {rig }}^{1}\left(X_{s}, U_{n+1}^{\vee}\right) .
\end{aligned}
$$

LEMMA 3.17. The connecting homomorphism $\delta$ is the identity.

Proof. By dualising, the extension

$$
0 \rightarrow U_{n}^{\vee} \rightarrow U_{n+1}^{\vee} \rightarrow \mathcal{O}_{X_{s}}^{\dagger} \otimes_{K(s)} H_{\text {rig }}^{1}\left(X_{s}, U_{n}^{\vee}\right) \rightarrow 0
$$

corresponds to the identity under the isomorphism

$$
\operatorname{Ext}_{\text {Isoc }^{\dagger}\left(X_{s} / K(s)\right)}\left(\mathcal{O}_{X_{s}}^{\dagger} \otimes_{K(s)} H_{\text {rig }}^{1}\left(X_{s}, U_{n}^{\vee}\right), U_{n}^{\vee}\right) \cong \operatorname{End}_{K(s)}\left(H_{\text {rig }}^{1}\left(X_{s}, U_{n}^{\vee}\right)\right)
$$

Now the Lemma follows from the fact that, for an extension $0 \rightarrow E \rightarrow F \rightarrow$ $\mathcal{O}_{X_{s}}^{\dagger} \otimes_{K(s)} V \rightarrow 0$ of a trivial bundle by $E$, the class of the extensions under the isomorphism

$$
\begin{aligned}
\operatorname{Ext}_{\mathrm{Isoc}^{\dagger}\left(X_{s} / K(s)\right)}\left(\mathcal{O}_{X_{s}}^{\dagger} \otimes_{K} V, E\right) & \cong V^{\vee} \otimes_{K(s)} H_{\text {rig }}^{1}\left(X_{s}, E\right) \\
& \cong \operatorname{Hom}_{K(s)}\left(V, H_{\text {rig }}^{1}\left(X_{s}, E\right)\right)
\end{aligned}
$$


is just the connecting homomorphism for the long exact sequence

$$
0 \rightarrow H_{\text {rig }}^{0}\left(X_{s}, E\right) \rightarrow H_{\text {rig }}^{0}\left(X_{s}, F\right) \rightarrow V \rightarrow H_{\text {rig }}^{1}\left(X_{s}, E\right) .
$$

In particular, any extension of $U_{n}$ by a trivial bundle $V \otimes_{K(s)} \mathcal{O}_{X_{s}}^{\dagger}$ is split after pulling back to $U_{n+1}$, and $H_{\text {rig }}^{0}\left(X_{s}, U_{n+1}^{\vee}\right) \cong H_{\mathrm{dR}}^{0}\left(X_{s}, U_{n}^{\vee}\right)$. It then follows by induction that $H_{\text {rig }}^{0}\left(X_{s}, U_{n}^{\vee}\right) \cong H_{\text {rig }}^{0}\left(X_{s}, \mathcal{O}_{X_{s}}^{\dagger}\right) \cong K(s)$ for all $n$.

Remarks 3.18. We can also show inductively, using Proposition 3.16, that each $U_{n+1}$ can be endowed with some Frobenius structure, since for any Frobenius structure on $U_{n}$, the identity in $\operatorname{End}_{K(s)}\left(H_{\text {rig }}^{1}\left(X_{s}, U_{n}^{\vee}\right)\right)$ will be Frobenius invariant, and hence will lift to some Frobenius structure on $U_{n+1}$.

Definition 3.19. Define the unipotent class of $E \in \mathcal{N} \operatorname{Isoc}^{\dagger}\left(X_{s} / K(s)\right)$ inductively as follows. If $E$ is trivial, then we say $E$ has unipotent class 1 . If there exists an extension

$$
0 \rightarrow V \otimes_{K(s)} \mathcal{O}_{X_{s}}^{\dagger} \rightarrow E \rightarrow E^{\prime} \rightarrow 0
$$

with $E^{\prime}$ of unipotent class $\leq m-1$, then we say that $E$ has unipotent class $\leq m$.

Now let $x=p(s), u_{1}=1 \in x^{*}\left(U_{1}\right)=K(s)$, and choose a compatible system of elements $u_{n} \in x^{*}\left(U_{n}\right)$ mapping to $u_{1}$.

Proposition 3.20. Let $F \in \mathcal{N} \operatorname{Isoc}^{\dagger}\left(X_{s} / K(s)\right)$ be an object of unipotent class $\leq m$. Then for all $n \geq m$ and any $f \in x^{*}(F)$ there exists a homomorphism $\alpha: U_{n} \rightarrow F$ such that $\left(x^{*} \alpha\right)\left(u_{n}\right)=f$.

Proof. As in the characteristic zero case, we copy the proof of Proposition 2.1.6 of [24] and use induction on $m$. The case $m=1$ is straightforward. For the inductive step, let $F$ be of unipotent class $m$, and choose an exact sequence

$$
0 \rightarrow E \stackrel{\psi}{\rightarrow} F \stackrel{\phi}{\rightarrow} G \rightarrow 0
$$

with $E$ trivial and $G$ of unipotent class $<m$. By induction there exists a morphism $\beta: U_{n-1} \rightarrow G$ such that $\left(x^{*} \phi\right)(f)=\left(x^{*} \beta\right)\left(u_{n-1}\right)$. Pulling back the extension (106) first by the morphism $\beta$ and then by the natural surjection $U_{n} \rightarrow U_{n-1}$ gives an extension of $U_{n}$ by $E$, which must split, as observed above.

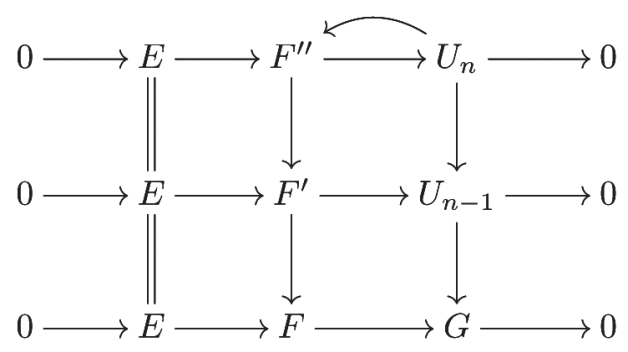


Let $\gamma: U_{n} \rightarrow F$ denote the induced morphism, then $\left(x^{*} \phi\right)\left(\left(x^{*} \gamma\right)\left(u_{n}\right)-f\right)=0$. Hence there exists some $e \in x^{*} E$ such that $\left(x^{*} \psi\right)(e)=\left(x^{*} \gamma\right)\left(u_{n}\right)-f$. Again by induction we can choose $\gamma^{\prime}: U_{n} \rightarrow E$ with $\left(x^{*} \gamma^{\prime}\right)\left(u_{n}\right)=e$. Finally let $\alpha=\gamma-\psi \circ \gamma^{\prime}$, it is easily seen that $\left(x^{*} \alpha\right)\left(u_{n}\right)=f$.

Corollary 3.21. Every $E$ in $\mathcal{N} \operatorname{Isoc}^{\dagger}\left(X_{s} / K(s)\right)$ is a quotient of $U_{n}^{\oplus m}$ for some $n, m \in \mathbb{N}$.

Recall that we have the higher direct images $\mathbf{R}^{i} g_{*}(E)$ for any $E \in F$-Isoc ${ }^{\dagger}(X / K)$. Thanks to 2.1.4 of [6], and the compatibilities already noted between tensor products and pull-backs of arithmetic $\mathcal{D}$-modules and their counterparts for overconvergent $F$-isocrystals, these satisfy a projection formula

$$
\mathbf{R}^{i} g_{*}\left(E \otimes g^{*} E^{\prime}\right) \cong \mathbf{R}^{i} g_{*}(E) \otimes E^{\prime}
$$

for any $E \in F$-Isoc ${ }^{\dagger}(X / K)$ and $E^{\prime} \in F$-Isoc ${ }^{\dagger}(S / K)$. If we let $h$ denote the structure morphism of $S$, then the fact that $h_{+} \circ g_{+}=(h \circ g)_{+}$implies that there is a Leray spectral sequence relating $\mathbf{R}^{i} h_{*}, \mathbf{R}^{j} g_{*}$ and $\mathbf{R}^{i+j}(h \circ g)_{*}$, the exact sequence of low degree terms of which reads

$$
0 \rightarrow H_{\text {rig }}^{1}\left(S, g_{*} E\right) \rightarrow H_{\text {rig }}^{1}(X, E) \rightarrow H_{\text {rig }}^{0}\left(S, \mathbf{R}^{1} g_{*} E\right) \rightarrow H_{\text {rig }}^{2}\left(S, g_{*} E\right) \rightarrow H_{\text {rig }}^{2}(X, E) .
$$

We are now in a position to inductively extend the $U_{n}$ to $X$. Let $W_{1}=\mathcal{O}_{X}^{\dagger}$.

THEOREM 3.22. There exists an extension $W_{n+1}$ of $W_{n}$ by $g^{*}\left(\mathbf{R}^{1} g_{*} W_{n}^{\vee}\right)^{\vee}$ in the category $\mathcal{N}_{g} F-\operatorname{Isoc}^{\dagger}(X / K)$ such that $i_{s}^{*} W_{n+1}=U_{n+1}$ and $g_{*} W_{n+1}^{\vee} \cong \mathcal{O}_{S}^{\dagger}$.

Proof. The statement and its proof are by induction on $n$, and in order to prove it we strengthen the induction hypothesis by also requiring that there exists a morphism $p^{*} W_{n}^{\vee} \rightarrow \mathcal{O}_{S}^{\dagger}$ such that the composite morphism $\mathcal{O}_{S}^{\dagger} \cong g_{*} W_{n}^{\vee} \cong p^{*} g^{*} g_{*} W_{n}^{\vee} \rightarrow$ $p^{*} W_{n}^{\vee} \rightarrow \mathcal{O}_{S}^{\dagger}$ is an isomorphism.

To check the base case we simply need to verify that $g_{*} \mathcal{O}_{X}^{\dagger} \cong \mathcal{O}_{S}^{\dagger}$. By the results of the previous section, we get a natural morphism $\mathcal{O}_{S}^{\dagger} \rightarrow g_{*} \mathcal{O}_{X}^{\dagger}$ as the unit of the adjunction between $g_{*}$ and $g^{*}$. By naturality, restricting this morphism to the fibre over $s$ gives us the unit $K(s) \rightarrow H_{\text {rig }}^{0}\left(X_{s}, \mathcal{O}_{X_{s}}^{\dagger}\right)$ of the adjunction between $H_{\text {rig }}^{0}\left(X_{s}, \cdot\right)$ and $\cdot \otimes_{K} \mathcal{O}_{X_{s}}^{\dagger}$, which is easily checked to be an isomorphism. Hence by rigidity, $\mathcal{O}_{S}^{\dagger} \rightarrow g_{*} \mathcal{O}_{X}^{\dagger}$ is an isomorphism.

So now suppose that we have $W_{n}$ as claimed. We look at the extension group

$$
\operatorname{Ext}_{F-\mathrm{Isoc}^{\dagger}(X / K)}\left(W_{n}, g^{*}\left(\mathbf{R}^{1} g_{*} W_{n}^{\vee}\right)^{\vee}\right) \rightarrow H_{\text {rig }}^{1}\left(X, W_{n}^{\vee} \otimes g^{*}\left(\mathbf{R}^{1} g_{*} W_{n}^{\vee}\right)^{\vee}\right)^{\phi=1} .
$$

The Leray spectral sequence, the projection formula above and the induction hypothesis that $g_{*} W_{n}^{\vee} \cong \mathcal{O}_{S}^{\dagger}$ give us an exact sequence 


$$
\begin{array}{r}
0 \rightarrow H_{\text {rig }}^{1}\left(S,\left(\mathbf{R}^{1} g_{*} W_{n}^{\vee}\right)^{\vee}\right) \\
\rightarrow H_{\text {rig }}^{1}\left(X, W_{n}^{\vee} \otimes g^{*}\left(\mathbf{R}^{1} g_{*} W_{n}^{\vee}\right)^{\vee}\right) \\
\rightarrow H_{\text {rig }}^{0}\left(S, \mathcal{E} \text { nd }\left(\mathbf{R}^{1} g_{*} W_{n}^{\vee}\right)\right) \rightarrow H_{\text {rig }}^{2}\left(S,\left(\mathbf{R}^{1} g_{*} W_{n}^{\vee}\right)^{\vee}\right) \\
\rightarrow H_{\text {rig }}^{2}\left(X, W_{n}^{\vee} \otimes g^{*}\left(\mathbf{R}^{1} g_{*} W_{n}^{\vee}\right)^{\vee}\right) .
\end{array}
$$

Now, the projection $p^{*} W_{n}^{\vee} \rightarrow \mathcal{O}_{S}^{\dagger}$ induces a map

$$
H_{\text {rig }}^{i}\left(S, p^{*} W_{n}^{\vee} \otimes\left(\mathbf{R}^{1} g_{*} W_{n}^{\vee}\right)^{\vee}\right) \rightarrow H_{\text {rig }}^{i}\left(S, \mathbf{R}^{1} g_{*}\left(W_{n}^{\vee}\right)^{\vee}\right)
$$

for $i=1,2$ such that the composite (dotted) arrow

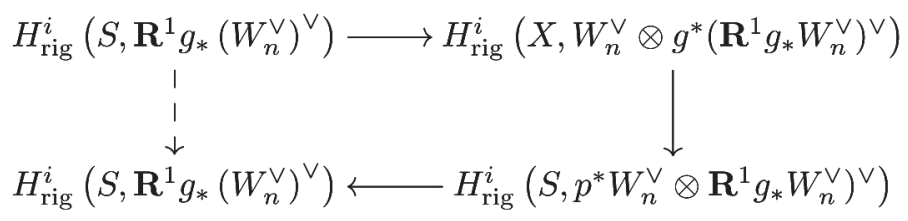

is an isomorphism, since it can be identified with the map induced by the composite arrow $\mathcal{O}_{S} \cong f_{*} W_{n}^{\vee} \cong p^{*} f^{*} f_{*} W_{n}^{\vee} \rightarrow p^{*} W_{n}^{\vee} \rightarrow \mathcal{O}_{S}$. Hence both the maps

$$
\begin{aligned}
& H_{\text {rig }}^{1}\left(S, \mathbf{R}^{1} g_{*}\left(W_{n}^{\vee}\right)^{\vee}\right) \rightarrow H_{\text {rig }}^{1}\left(X, W_{n}^{\vee} \otimes g^{*}\left(\mathbf{R}^{1} g_{*} W_{n}^{\vee}\right)^{\vee}\right) \\
& H_{\text {rig }}^{2}\left(S, \mathbf{R}^{1} g_{*}\left(W_{n}^{\vee}\right)^{\vee}\right) \rightarrow H_{\text {rig }}^{2}\left(X, W_{n}^{\vee} \otimes g^{*}\left(\mathbf{R}^{1} g_{*} W_{n}^{\vee}\right)^{\vee}\right)
\end{aligned}
$$

appearing in the 5 -term exact sequence split, compatibly with Frobenius actions. So there is a commutative diagram

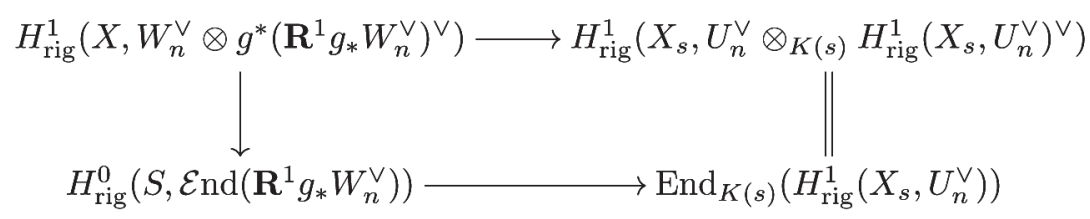

where the horizontal arrows are just restrictions to the fibre over $s$, and the left hand vertical arrow is surjective. The identity morphism in $\operatorname{End}_{K(s)}\left(H_{\text {rig }}^{1}\left(X_{s}, U_{n}^{\vee}\right)\right)$, which is Frobenius invariant (for some Frobenius structure on $U_{n}$, see Remark 3.18) and corresponds to the extension $U_{n+1}$, lifts to the identity in

$$
H_{\mathrm{rig}}^{0}\left(S, \mathcal{E} \operatorname{nd}\left(\mathbf{R}^{1} g_{*} W_{n}^{\vee}\right)\right)=\operatorname{End}_{\mathrm{Isoc}^{\dagger}(S / K)}\left(\mathbf{R}^{1} g_{*} W_{n}^{\vee}\right),
$$

and this element is also Frobenius invariant. Thus there exists a unique Frobenius invariant element of $H_{\text {rig }}^{1}\left(X, W_{n}^{\vee} \otimes g^{*}\left(\mathbf{R}^{1} g_{*} W_{n}^{\vee}\right)^{\vee}\right)$ lifting the identity in $\operatorname{End}_{I^{\prime}{ }^{\dagger}(S / K)}\left(\mathbf{R}^{1} g_{*} W_{n}^{\vee}\right)$, and which maps to zero under the above splitting

$$
H_{\text {rig }}^{1}\left(X, W_{n}^{\vee} \otimes g^{*}\left(\mathbf{R}^{1} g_{*} W_{n}^{\vee}\right)^{\vee}\right) \rightarrow H_{\text {rig }}^{1}\left(S, \mathbf{R}^{1} g_{*}\left(W_{n}^{\vee}\right)^{\vee}\right) .
$$


Let $W_{n+1}^{\prime}$ be any corresponding extension (the map from the extension group as $F$-isocrystals to the Frobenius invariant part of $H^{1}$ is surjective). Now, we have a natural map

$$
\operatorname{Ext}_{F-\operatorname{Isoc}^{\dagger}(S / K)}\left(\mathcal{O}_{S}^{\dagger},\left(\mathbf{R}^{1} g_{*} W_{n}^{\vee}\right)^{\vee}\right) \stackrel{g^{*}}{\rightarrow} \operatorname{Ext}_{F-\operatorname{Isoc}^{\dagger}(X / K)}\left(W_{n}, g^{*}\left(\mathbf{R}^{1} g_{*} W_{n}^{\vee}\right)^{\vee}\right)
$$

which has a section (denoted $p^{*}$ ) induced by the map $p^{*} W_{n}^{\vee} \rightarrow \mathcal{O}_{S}^{\dagger}$, and such that the whole diagram

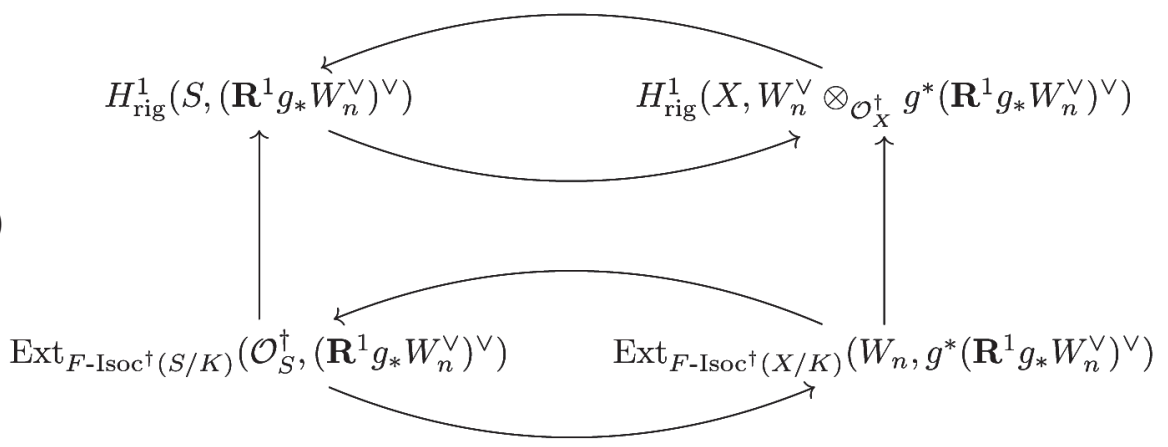

commutes. We let $W_{n+1}$ be the extension corresponding to $\left[W_{n+1}^{\prime}\right]-g^{*} p^{*}\left[W_{n+1}^{\prime}\right]$ in

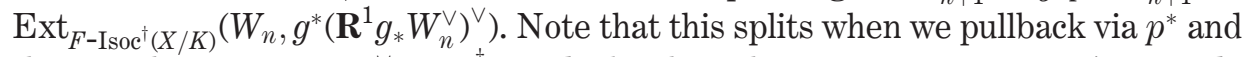
then push-out via $p^{*} W_{n}^{\vee} \rightarrow \mathcal{O}_{S}^{\dagger}$, and also has the same image as $W_{n+1}^{\prime}$ inside $H_{\text {rig }}^{1}\left(X, W_{n}^{\vee} \otimes_{\mathcal{O}_{X}^{\dagger}} g^{*}\left(\mathbf{R}^{1} g_{*} W_{n}^{\vee}\right)^{\vee}\right)$.

To complete the induction we need to show that $g_{*} W_{n+1}^{\vee} \cong \mathcal{O}_{S}^{\dagger}$, and that there exists a map $p_{*} W_{n+1}^{\vee} \rightarrow \mathcal{O}_{S}^{\dagger}$ as claimed. We have an exact sequence (using the projection formula and the fact that $g_{*} \mathcal{O}_{X}^{\dagger} \cong \mathcal{O}_{S}^{\dagger}$ )

$$
0 \rightarrow g_{*} W_{n}^{\vee} \rightarrow g_{*} W_{n+1}^{\vee} \rightarrow \mathbf{R}^{1} g_{*} W_{n}^{\vee} \rightarrow \ldots
$$

and it follows from Lemma 3.17 together with base change that the arrow $g_{*} W_{n}^{\vee} \rightarrow g_{*} W_{n+1}^{\vee}$ restricts to an isomorphism on the fibre at $s$. Thus by rigidity it is an isomorphism. Finally, we have an exact sequence

$$
0 \rightarrow p^{*} W_{n}^{\vee} \rightarrow p^{*} W_{n+1} \rightarrow\left(\mathbf{R}^{1} g_{*} W_{n}^{\vee}\right)^{\vee} \rightarrow 0
$$

which splits when we push-out via the map $p^{*} W_{n}^{\vee} \rightarrow \mathcal{O}_{S}^{\dagger}$. This splitting induces a $\operatorname{map} p^{*} W_{n+1}^{\vee} \rightarrow \mathcal{O}_{S}^{\dagger}$ such that the diagram

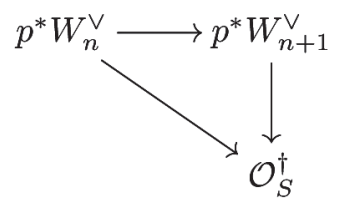


commutes. Now the fact that the diagram

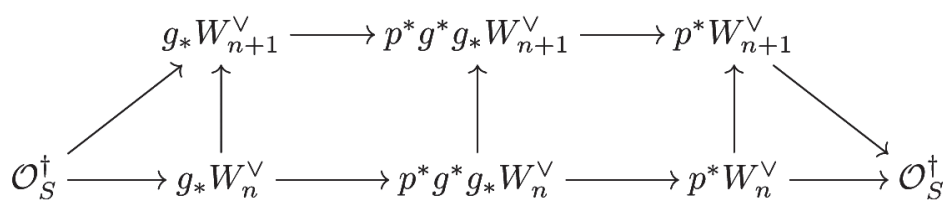

commutes implies that the composite along the top row is an isomorphism, finishing the proof.

To complete the proof of Theorem 3.7, we use the base extension functor

$$
-\otimes_{K} K(s): \mathcal{N}_{g} F-\operatorname{Isoc}^{\dagger}(X / K) \rightarrow \mathcal{N}_{g} F-\operatorname{Isoc}^{\dagger}(X / K) \otimes_{K} K(s),
$$

which is defined on pages 155-156 of [31], to view the $W_{n}$ as objects of the latter category.

\section{2 - Frobenius structures}

The upshot of the previous section is that we now have an affine group scheme $\pi_{1}^{\text {rig }}(X / S, p)$ over the Tannakian category $F$-Isoc ${ }^{\dagger}(S / K)$ whose fibre (ignoring Frobenius structures) over any closed point $s$ is the usual rigid fundamental group $\pi_{1}^{\text {rig }}\left(X_{s}, p_{s}\right)$ as defined by Chiarellotto and Le Stum in [16]. In Chapter II of [15], Chiarellotto defines a Frobenius isomorphism $F_{*}: \pi_{1}^{\text {rig }}\left(X_{s}, p_{s}\right) \stackrel{\sim}{\rightarrow} \pi_{1}^{\text {rig }}\left(X_{s}, p_{s}\right)$, by using the fact that Frobenius pullback induces an automorphism of the category $\mathcal{N} \operatorname{Isoc}^{\dagger}\left(X_{s} / K\right)$. Since we have constructed $\pi_{1}^{\mathrm{rig}}(X / S, p)$ as an affine group scheme over $F$-Isoc ${ }^{\dagger}(S / K)$, it comes with a Frobenius structure that we can compare with Chiarellotto's. However, it is not obvious to us exactly what the relationship between these two Frobenius structures is, so instead we will endow $\pi_{1}^{\mathrm{rig}}(X / S, p)$ with a different Frobenius, which we will be able to compare with the natural Frobenius on the fibres.

REMARKS 3.23. From now onward, we will consider $\pi_{1}^{\text {rig }}(X / S, p)$ as an affine group scheme over Isoc ${ }^{\dagger}(S / K)$, via the forgetful functor.

Let $\sigma_{S}: S \rightarrow S$ denote the $k$-linear Frobenius, $X^{\prime}=X \times_{S, \sigma_{S}} S$ the base change of $X$ by $\sigma_{S}$, and $\sigma_{X / S}: X \rightarrow X^{\prime}$ the relative Frobenius induced by the $k$-linear Frobenius $\sigma_{X}$ of $X$. Let $p^{\prime}$ be the induced point of $X^{\prime}$, and $q=\sigma_{X / S} \circ p \in X^{\prime}(S)$. Then by functoriality and base change we get a homomorphism

$$
\pi_{1}^{\mathrm{rig}}(X / S, p) \rightarrow \pi_{1}^{\mathrm{rig}}\left(X^{\prime} / S, q\right)
$$

and an isomorphism

$$
\pi_{1}^{\mathrm{rig}}\left(X^{\prime} / S, p^{\prime}\right) \stackrel{\sim}{\rightarrow} \sigma_{S}^{*} \pi_{1}^{\mathrm{rig}}(X / S, p) .
$$


One can easily check that $p^{\prime}=q \in X(S)$, and hence we get a natural morphism $\phi: \pi_{1}^{\mathrm{rig}}(X / S, p) \rightarrow \sigma_{S}^{*} \pi_{1}^{\mathrm{rig}}(X / S, p)$.

\section{LEMMA 3.24. This is an isomorphism.}

Proof. Let $s \in S$ be a closed point, with residue field $k(s)$ of size $q^{a}$. The map induced by $\phi^{a}$ on the fibre $\pi_{1}^{\text {rig }}\left(X_{s}, p_{s}\right)$ over $s$ is the same as that induced by pulling back unipotent isocrystals on $X_{s}$ by the $k(s)$-linear Frobenius on $X_{s}$. This is proved in Chapter II of [15] to be an isomorphism, thus $\phi^{a}$ is an isomorphism by rigidity. Hence $\phi$ is also an isomorphism.

We now let $F_{*}: \sigma_{S}^{*} \pi_{1}^{\text {rig }}(X / S, p) \stackrel{\sim}{\rightarrow} \pi_{1}^{\text {rig }}(X / S, p)$ denote the inverse of $\phi$, which by the proof of the previous lemma, reduces to the Frobenius structure as defined by Chiarellotto on closed fibres.

Definition 3.25. When we refer to 'the' Frobenius on $\pi_{1}^{\text {rig }}(X / S, p)$, we will mean the isomorphism $F_{*}$ just defined.

\section{3 - Cohomology and period maps}

In this section we study the non-abelian cohomology of the unipotent quotients $\pi_{1}^{\mathrm{rig}}(X / S, p)_{n}$ of $\pi_{1}^{\mathrm{rig}}(X / S, p)$. Assumptions and notations will be exactly as in the previous two sections, so $g: X \rightarrow S$ will be a 'good', realisable morphism of varieties over a finite field, and $p$ a section. Recall from Section 2.1 the notion of a torsor under an affine group scheme $U$ over $\operatorname{Isoc}^{\dagger}(S / K)$.

Definition 3.26. We define $H_{\text {rig }}^{1}(S, U)$ to be the pointed set of isomorphism classes of torsors under $U$.

ExAmple 3.27. Suppose that $U$ is the vector scheme associated to an overconvergent isocrystal $E$. Then Example 5.10 of [19] shows that there is a bijection $H_{\text {rig }}^{1}(S, U) \stackrel{\sim}{\rightarrow} H_{\text {rig }}^{1}(S, E)$.

If $U$ has a Frobenius structure, that is an isomorphism $\phi: \sigma_{S}^{*} U \stackrel{\sim}{\rightarrow} U$, then we can define an $F$-torsor under $U$ to be a $U$-torsor $P$, together with a Frobenius isomorphism $\phi_{P}: \sigma_{S}^{*} P \stackrel{\sim}{\rightarrow} P$ such that the action map $P \times U \rightarrow P$ is compatible with Frobenius.

Definition 3.28. We define $H_{F, \text { rig }}^{1}(S, U)$ to be the set of isomorphism classes of $F$-torsors under $U$.

Given any torsor $P$ under $U$, without $F$-structure, $\sigma_{S}^{*} P$ will be a torsor under $\sigma_{S}^{*} U$, and hence we can use the isomorphism $\phi$ to consider $\sigma_{S}^{*} P$ as a torsor under $U$. 
Hence we get a Frobenius action $\phi: H_{\text {rig }}^{1}(S, U) \rightarrow H_{\text {rig }}^{1}(S, U)$, and it is easy to see that the forgetful map

$$
H_{F, \text { rig }}^{1}(S, U) \rightarrow H_{\text {rig }}^{1}(S, U)
$$

is a surjection onto the subset $H_{\text {rig }}^{1}(S, U)^{\phi=\text { id }}$ fixed by the action of $\phi$.

Given any point $x \in X(S)$, we have the path torsors $P(x)$ under $\pi_{1}^{\text {rig }}(X / S, p)$ as well as the finite level versions $P(x)_{n}$. Moreover, these come with Frobenius structures, and hence we get compatible maps

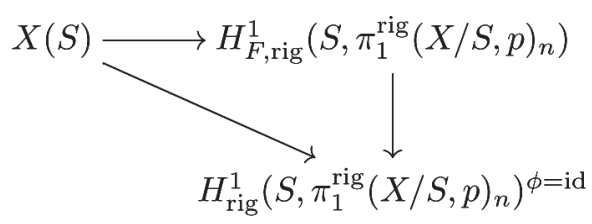

for each $n \geq 1$.

In order to get a handle on this 'non-abelian' $H^{1}$, we first discuss the generalisation of Theorem 2.11 of [31] to non-neutral Tannakian categories via groupoids and their representations, following [20]. The reason for doing this is to obtain a generalisation of Example 2.2 giving a more explicit description of $H_{\text {rig }}^{1}(S, U)$.

So let $K$ be a field of characteristic 0 , and $Y$ a $K$-scheme.

Definition 3.29. A $K$-groupoid acting on $Y$ is a $K$-scheme $G$, together with 'source' and 'target' morphisms $s, t: G \rightarrow Y$ and a 'law of composition' $\circ: G \times \times_{s^{t}} G \rightarrow G$ such that the diagram

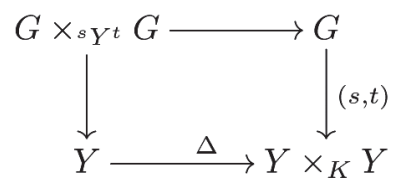

commutes, and such that the following condition holds: for any $K$-scheme $T$, the data of $Y(T), G(T), s, t, \circ$ forms a groupoid, where $Y(T)$ is the set of objects and $G(T)$ the set of morphisms.

ExamPle 3.30. Suppose that $Y=\operatorname{Spec} K$. Then a $K$-groupoid acting on $Y$ is just a group scheme over $K$.

DeFINITION 3.31. If $G$ is a $K$-groupoid acting on $Y$, then a representation of $G$ is a quasi-coherent $\mathcal{O}_{Y}$-module $V$, together with a morphism $\rho(g): s(g)^{*} V \rightarrow t(g)^{*} V$ for any $K$-scheme $T$ and any point $g \in G(T)$. These morphisms must be compatible with base change $T^{\prime} \rightarrow T$, as well as with the law of composition on $G$. Finally, if $\operatorname{id}_{y} \in G(T)$ is the 'identity morphism' corresponding to the 'object' $y \in Y(T)$, then we require the morphism $\rho\left(\mathrm{id}_{y}\right)$ to be the identity. A morphism of representations is 
defined in the obvious way, and we denote the category of coherent representations by $\operatorname{Rep}(Y: G)$. Of course we can similarly define actions of $G$ on any (group) scheme $U$ over $Y$, by instead requiring morphisms $\rho(g): U \times_{Y, s(g)} T \rightarrow U \times_{Y, t(g)} T$ of (group) schemes over $T$.

EXAMPLE 3.32. If $Y=$ Spec $K$, then this just boils down to the usual definition of a representation of a group scheme over $K$.

Now suppose that $\mathcal{C}$ is a Tannakian category over $K$, which admits a fibre functor $\omega: \mathcal{C} \rightarrow \operatorname{Vec}_{L}$ taking values in some finite extension $L / K$. Let $\operatorname{pr}_{i}: \operatorname{Spec}\left(L \otimes_{K} L\right) \rightarrow \operatorname{Spec}(L)$ for $i=1,2$ denote the two projections. Then we get two fibre functors $\operatorname{pr}_{i}^{*} \circ \omega: \mathcal{C} \rightarrow \operatorname{Mod}_{\text {f.g. }}\left(L \otimes_{K} L\right)$ taking values in the category of finitely generated $L \otimes_{K} L$-modules, and the functor of isomorphisms $\underline{\mathrm{Isom}}^{\otimes}\left(\mathrm{pr}_{1}^{*} \circ \omega, \mathrm{pr}_{2}^{*} \circ \omega\right)$ is represented by an affine scheme $\underline{\mathrm{Aut}}_{K}^{\otimes}(\omega)$ over $L \otimes_{K} L$. The composite of the map $\underline{\operatorname{Aut}}_{K}^{\otimes}(\omega) \rightarrow \operatorname{Spec}\left(L \otimes_{K} L\right)$ with the two projections to $\operatorname{Spec}(L)$ makes $\operatorname{Aut}_{K}^{\otimes}(\omega)$ into a $K$-groupoid acting on $\operatorname{Spec}(L)$. Moreover, if $E$ is an object of $\mathcal{C}$, then $\omega(E)$ becomes a representation of $\mathrm{Aut}_{K}^{\otimes}(\omega)$ in the obvious way. Thus we get a functor

$$
\mathcal{C} \rightarrow \operatorname{Rep}\left(L: \underline{\operatorname{Aut}}_{K}^{\otimes}(\omega)\right)
$$

and Théorème (1.12) of [20] states (in particular) the following.

THEOREM 3.33. The induced functor $\mathcal{C} \rightarrow \operatorname{Rep}\left(L: \underline{\operatorname{Aut}}_{K}^{\otimes}(\omega)\right)$ is an equivalence of Tannakian categories.

Finally, to get the generalisation of Example 2.2 that we need, the following technical lemma is necessary.

Lemma 3.34 ([20], Corollaire 3.9). Let $L / K$ be finite, and $G$ a K-groupoid acting on $\operatorname{Spec}(L)$, affine and faithfully flat over $L \otimes_{K} L$. Then any representation $V$ of $G$ is the colimit of its finite dimensional sub-representations.

CoRollary 3.35. If $\mathcal{C}$ is a Tannakian category over $K, \omega$ a fibre functor with values in $L$, then an affine (group) scheme over C 'is' just an affine (group) scheme over $L$ together with an action of $\underline{\mathrm{Aut}}_{K}^{\otimes}(\omega)$, and morphism of such objects 'are' just $\underline{\text { Aut }}_{K}^{\otimes}(\omega)$-equivariant morphisms.

Definition 3.36. Let $G$ be a $K$-groupoid acting on $\operatorname{Spec}(L)$. If $U$ is a group scheme over $L$ with a $G$-action, we will denote by $H^{1}(G, U)$ the set of isomorphism classes of $G$-equivariant torsors under $U$.

EXAMPLE 3.37. - If $V$ is a representation of $G$, then $\operatorname{Spec}\left(\operatorname{Sym}\left(V^{\vee}\right)\right)$ naturally becomes a group scheme over $L$ with a $G$-action. We will refer to this latter object as the vector scheme associated to $V$. 
- If $U$ is a unipotent affine group scheme over Isoc ${ }^{\dagger}(S / K)$ as above, then for any closed point $s \in S$, the unipotent group $U_{s}$ over $K(s)$ attains an action of the $K$-groupoid $\underline{\mathrm{Aut}}_{K}^{\otimes}\left(s^{*}\right)$, and there is a natural bijection of sets

$$
H_{\text {rig }}^{1}(S, U) \stackrel{\sim}{\rightarrow} H^{1}\left(\underline{\mathrm{Aut}}_{K}^{\otimes}\left(s^{*}\right), U_{s}\right) .
$$

Suppose that $Y=\operatorname{Spec}(L)$, with $L / K$ finite, and let $G$ be a $K$ groupoid acting on $Y$. Let $U$ be a unipotent group over $L$, on which $G$ acts.

Definition 3.38. A 1-cocyle for $G$ with values in $U$ is a map of $K$-schemes $\phi: G \rightarrow U$ such that

- The diagram

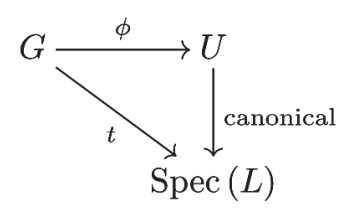

commutes.

- For any $K$-scheme $T$, and points $g, h \in G(T)$ which are composable in the sense that $s(g)=t(h), \phi(g h)=\phi(g) \cdot \rho(g)(\phi(h))$ holds. This equality needs some explanation. By the first condition above, $\phi(g)$ lands in the subset $\operatorname{Hom}_{T}\left(T, U \times_{L, t(g)} T\right)$ of $\operatorname{Hom}_{K}(T, U)$ which consists of those morphisms $T \rightarrow U$ which are such that the diagram

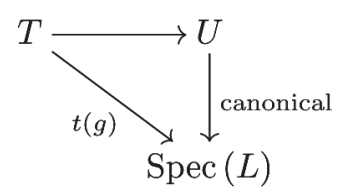

commutes. Similarly, $\phi(h) \in \operatorname{Hom}_{T}\left(T, U \times_{L, t(h)} T\right)=\operatorname{Hom}_{T}\left(T, U \times_{L, s(g)} T\right)$. Since $U / L$ is a group scheme, $\operatorname{Hom}_{T}\left(T, U \times_{L, t(g)} T\right)$ is a group, and the action of $G$ on $U$ gives a homomorphism

$$
\rho(g): \operatorname{Hom}_{T}\left(T, U \times_{L, s(g)} T\right) \rightarrow \operatorname{Hom}_{T}\left(T, U \times_{L, t(g)} T\right) .
$$

Hence the equality $\phi(g h)=\phi(g) \cdot \rho(g)(\phi(h))$ makes sense inside the group $\operatorname{Hom}_{T}\left(T, U \times_{L, t(g)} T\right)$.

The set of 1-cocycles with coefficients in $U$ is denoted $Z^{1}(G, U)$. This set has a natural action of $U(L)$ via

$$
(\phi * u)(g)=\left(t(g)^{*} u\right)^{-1} \cdot \phi(g) \cdot \rho(g)\left(s(g)^{*}(u)\right)
$$

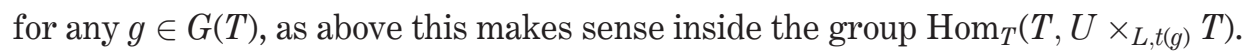

The point of introducing these definitions is the following. 
Lemma 3.39. Let $G$ be a K-groupoid acting on $\operatorname{Spec}(L)$, and $U$ a pro-unipotent group scheme over $L$ with a G-action. Then there is a bijection between the non-abelian cohomology set $H^{1}(G, U)$ and the set of orbits of $Z^{1}(G, U)$ under the action of $U(L)$.

Proof. Let $P$ be a $G$-equivariant torsor under $U$. Since any torsor under a unipotent group scheme over an affine scheme is trivial, we may choose a point $p \in P(L)$. Now, for any $g \in G(T)$ we can consider the points $t(g)^{*} p$ and $s(g)^{*} p$ inside $\operatorname{Hom}_{T}\left(T, P \times_{L, t(g)} T\right)$ and $\operatorname{Hom}_{T}\left(T, P \times_{L, s(g)} T\right)$ respectively. We have a morphism $\rho(g): P \times_{L, s(g)} T \rightarrow P \times_{L, t(g)} T$ and hence there exists a unique element $\phi(g) \in$ $U \times_{L, t(g)} T(T)$ such that $t(g)^{*} p \phi(g)=\rho(g) s(g)^{*} p$. Thus we get some $\phi(g) \in U(T)$, and the map $g \mapsto \phi(g)$ is functorial, giving a map of schemes $\phi: G \rightarrow U$. The fact that $\phi(g) \in \operatorname{Hom}_{T}\left(T, U \times_{L, t(g)} T\right)$ means that the diagram

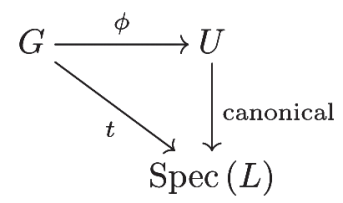

commutes, and one easily checks that $\phi$ satisfies the cocycle condition. A different choice of $p$ differs by an element of $U(L)$, and one easily sees that this modifies $\phi$ exactly as in the action of $U(L)$ on $Z^{1}(G, U)$ defined above. Hence we get a well defined map

$$
H^{1}(G, U) \rightarrow Z^{1}(G, U) / U(L) .
$$

Conversely, given a cocycle $\phi: G \rightarrow U$, we can define a torsor $P$ as follows. The underlying scheme of $P$ is just $U$, and the action of $U$ on $P$ is just the usual action of right multiplication. We use the cocycle $\phi$ to twist the action of $G$ as follows. If $g \in G(T)$, then we define $\rho(g): P \times_{L, s(g)} T \rightarrow P \times_{L, t(g)} T$ to be the unique map, compatible with the $U$ action, taking the identity of $U \times_{L, s(g)} T=$ $P \times_{L, s(g)} T$ to $\phi(g) \in U \times_{L, t(g)} T=P \times_{L, t(g)} T$. One easily checks that this descends to the quotient $Z^{1}(G, U) / U(L)$, and provides an inverse to the map defined above.

We now want to investigate more closely the case when $U$ is a vector scheme, coming from some finite dimensional representation $V$ of $G$. In this case we define, for any $n \geq 0$ the space $C^{n}(G, V)$ of $n$-cochains of $G$ in $V$ as follows. Let $G^{(n)}$ denote the scheme of ' $n$-fold composable arrows in $G$ ', that is the sub-scheme of $G \times \times_{K} \ldots \times_{K} G$ ( $n$ copies), consisting of those points $\left(g_{1}, \ldots, g_{n}\right)$ such that $s\left(g_{i}\right)=t\left(g_{i+1}\right)$ for all $i$, by convention we set $G^{(0)}=\operatorname{Spec}(L)$. Then the space of $n$ cochains is simply the space of global sections of the coherent sheaf $\left(\delta_{1}^{n}\right)^{*} V$ on $G^{(n)}$, where $\delta_{1}^{n}: G^{(n)} \rightarrow \operatorname{Spec}(L)$ is defined to be the map $t \circ \operatorname{pr}_{1}$, where $\operatorname{pr}_{1}: G^{n} \rightarrow G$ is projection onto the first factor. This can also be viewed as the set of morphisms 
$G^{(n)} \rightarrow \operatorname{Spec}\left(\operatorname{Sym}\left(V^{\vee}\right)\right)$ making the diagram

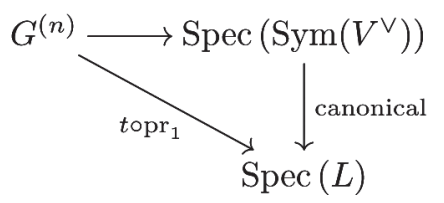

commute, and hence we can define differentials $d^{n}: C^{n}(G, V) \rightarrow C^{n+1}(G, V)$ by

$$
\begin{gathered}
\left(d^{n} \phi\right)\left(g_{1}, \ldots, g_{n+1}\right)=\rho\left(g_{1}\right) \phi\left(g_{2}, \ldots, g_{n+1}\right) \\
+\sum_{i=1}^{n}(-1)^{i} \phi\left(g_{1}, \ldots, g_{i} g_{i+1}, \ldots, g_{n+1}\right) \\
+(-1)^{n+1} \phi\left(g_{1}, \ldots, g_{n}\right)
\end{gathered}
$$

for $n \geq 1$, where $g_{1}, \ldots, g_{n+1}$ are composable elements of $G(T)$, and all the summands on the RHS are global sections of the coherent sheaf $t\left(g_{1}\right)^{*} V$ on $T$. For $n=0$ we define $\left(d^{0} \phi\right)(g)=\rho(g) \phi(s(g))-\phi(t(g))$. It is easily checked that these differentials make $C^{\bullet}(G, V)$ into a chain complex, and we define the cohomology of $G$ with coefficients in $V$ to be the cohomology of this complex:

$$
H^{n}(G, V):=H^{n}\left(C^{\bullet}(G, V)\right) \text {. }
$$

LEMma 3.40. Let $V$ be a representation of the groupoid $G$ acting on $\operatorname{Spec}(L)$. Then there is a canonical bijection $H^{1}(G, V) \stackrel{\sim}{\rightarrow} H^{1}\left(G\right.$, $\left.\operatorname{Spec}\left(\operatorname{Sym}\left(V^{\vee}\right)\right)\right)$.

Proof. Taking into account the description of the latter in terms of cocyles modulo the action of $V$, this is straightforward algebra.

So far we have been working over a field $K$, however, exactly the same definitions make sense over any $K$-algebra $R$, and we can similarly define the cohomology of an $R$-groupoid acting on $\operatorname{Spec}\left(R \otimes_{K} L\right)$. There is an obvious base extension functor, taking $K$-groupoids to $R$-groupoids, and hence we can define cohomology functors

$$
\underline{H}^{n}(G, V)(R)=H^{n}\left(G_{R}, V_{R}\right)
$$

for any representation $V$ of $G$. These are the cohomology groups of a natural complex-valued functor

$$
\underline{C}^{n}(G, V)(R)=C^{n}\left(G_{R}, V_{R}\right) .
$$

We will also denote by $\underline{Z}^{n}(G, V)$ and $\underline{B}^{n}(G, V)$ the 'n-cocycle' and ' $n$-coboundary' functors respectively.

Proposition 3.41. Suppose that $G=\operatorname{Spec}(A)$ is affine. Then for any K-algebra there are a canonical isomorphisms $H^{n}\left(G_{R}, V_{R}\right) \stackrel{\sim}{\rightarrow} H^{n}(G, V) \otimes_{K} R$ for all $n \geq 0$. 
Proof. In this case, there is an alternative algebraic description of the complex $C^{\bullet}(G, V)$. First of all, $A$ is a commutative $L \otimes_{K} L$-algebra, hence $A$ becomes an $L$ module in two different ways, using the two maps $L \rightarrow L \otimes_{K} L$. We will refer to these as the 'left' and 'right' structures, these two different $L$-module structures induce the same $K$-module structure. The groupoid structure corresponds to a morphism $\Delta: A \rightarrow A \otimes_{L} A$, using the two different $L$-module structures to form the tensor product.

The action of $G$ on a representation $V$ can be described by an $L$-linear map $\Delta_{V}: V \rightarrow V \otimes_{L, t} A$, where on the RHS we use the 'left' $L$-module structure on $A$ to form the tensor product, and define the $L$-module structure on the result via the 'right' $L$-module structure on $A$. This map is required to satisfy axioms analogous to the comodule axioms for the description of a representation of an affine group scheme.

Hence the group $C^{n}(G, V)$ of $n$-cochains is simply the $L$-module $V \otimes_{L} A \otimes_{L} \ldots \otimes_{L} A$ ( $n$ copies of $A$ ). We can describe the boundary maps $d^{n}$ algebraically as well by

$$
\begin{aligned}
d^{n}\left(v \otimes a_{1} \otimes \ldots \otimes a_{n}\right)= & \Delta_{V}(v) \otimes a_{1} \otimes \ldots \otimes a_{n} \\
& +\sum_{i=1}^{n} v \otimes a_{1} \otimes \ldots \otimes \Delta\left(a_{i}\right) \otimes \ldots \otimes a_{n} \\
& +v \otimes a_{1} \otimes \ldots \otimes a_{n} \otimes 1 .
\end{aligned}
$$

Exactly the same discussion applies over any $K$-algebra $R$, and one immediately sees that there is an isomorphism of complexes $C^{\bullet}\left(G_{R}, V_{R}\right) \cong C^{\bullet}(G, V) \otimes_{K} R$. Since any $K$-algebra is flat, the result follows.

REMARKS 3.42. In other words, the cohomology functor $\underline{H}^{n}(G, V)$ is represented by the vector scheme associated to $H^{n}(G, V)$.

If $U$ is a unipotent group scheme on which $G$ acts, we can also extend the sets $Z^{1}(G, U)$ and $H^{1}(G, U)$ to functors of $K$-algebras in the same way. We can also define $H^{0}(G, U)$ to be the group of all $u \in U(L)$ such that $\rho(g) s(g)^{*} u=t(g)^{*} u$ for any $g \in G(T)$, and any $K$-scheme $T$. We will also need to make use of a truncated cochain complex functor for non-abelian cohomology. This is the complex

$$
C^{0}(G, U) \rightarrow C^{1}(G, U) \rightarrow C^{2}(G, U)
$$

where $C^{n}(G, U)$ is the pointed set of maps $G^{(n)} \rightarrow U$ commuting with the projection to $\operatorname{Spec}(L), G^{(n)}$ being the scheme of $n$-fold composable arrows in $G$. Thus we can identify $C^{0}(G, U)$ with $U(L)$, and the differential $d^{0}: U(L) \rightarrow C^{1}(G, U)$ is defined by $d^{0} u(g)=\left(\rho(g) s(g)^{*} u\right)\left(t(g)^{*} u\right)^{-1}$ for any $g \in G(T)$. The differential $d^{1}: C^{1}(G, U) \rightarrow$ $C^{2}(G, U)$ is defined by

$$
d^{1} \phi(g, h)=\phi(g)(\rho(g) \phi(h)) \phi(g h)^{-1} .
$$


for $g \in G(T)$. Thus if we let $e_{1}, e_{2} \in C^{1}(G, U), C^{2}(G, U)$ denote the 'trivial' cochains then we get natural identifications $H^{0}(G, U)=\left(d^{0}\right)^{-1}\left(e_{1}\right)$ and $Z^{1}(G, U)=\left(d^{1}\right)^{-1}\left(e_{2}\right)$.

These constructions extend to functors $\underline{C}^{\bullet}(G, U), \underline{H}^{0}(G, U)$ of $K$-algebras in the obvious way. It is straightforward to check that when $V$ is a representation of $G$, $\underline{C}^{\bullet}\left(G, \operatorname{Spec}\left(\operatorname{Sym}\left(V^{\vee}\right)\right)\right)$ agrees with $\underline{C}^{\bullet}(G, V)$ in degrees $\leq 2$.

Proposition 3.43. Let $0 \rightarrow V \rightarrow U \rightarrow W \rightarrow 0$ be an exact sequence of unipotent group schemes over $K$, acted on by $G$, with $V$ a vector scheme. Suppose that $V$ is central in $W$. Then there is a sequence of cohomology functors

$$
\begin{aligned}
0 & \rightarrow \underline{H}^{0}(G, V) \rightarrow \underline{H}^{0}(G, U) \rightarrow \underline{H}^{0}(G, W) \rightarrow \underline{H}^{1}(G, V) \\
& \rightarrow \underline{H}^{1}(G, U) \rightarrow \underline{H}^{1}(G, W) \rightarrow \underline{H}^{2}(G, V)
\end{aligned}
$$

which is exact in the sense that for all $K$-algebras $R$, the induced sequence on $R$ points is exact as a sequence of pointed sets.

Proof. The only non-obvious part of the existence of the sequence is the construction and functoriality of the boundary maps $H^{i}\left(G_{R}, W_{R}\right) \rightarrow H^{i+1}\left(G_{R}, V_{R}\right)$, for $i=0,1$. So suppose that we're given a map $\phi: G_{R}^{(i)} \rightarrow W_{R}$ for $i=0,1$, which is a cocycle. Then we may lift this to a map $\tilde{\phi}: G_{R}^{(i)} \rightarrow U_{R}$, (since the surjection $U \rightarrow W$ always admits a section as a map of schemes) and we thus obtain a map $d^{i}(\tilde{\phi}): G_{R}^{(i)} \rightarrow V_{R}$ measuring the failure of this lifted cochain $\tilde{c}$ to be a cocycle. Then exactly as in Chapter VII, Appendix of [33], we can verify that $d^{i}(\tilde{c})$ is actually a cocycle, and the class of $d^{i}(\tilde{\phi})$ in $H^{i+1}\left(G_{R}, V_{R}\right)$ does not depend on either the class of the cocycle $\phi$ or on the lift $\tilde{\phi}$. It is also easy to check functoriality of the induced map $H^{i}\left(G_{R}, W_{R}\right) \rightarrow H^{i+1}\left(G_{R}, V_{R}\right)$, since a lifting of $\phi: G_{R}^{(i)} \rightarrow W_{R}$ to a map $\tilde{\phi}: G_{R}^{(i)} \rightarrow U_{R}$ then induces a lifting of $\phi_{S}: G_{S}^{(i)} \rightarrow W_{S}$ to $\tilde{\phi}_{S}: G_{S}^{(i)} \rightarrow U_{S}$ for any $R$-algebra $S$. That the sequence is exact on $R$-points is more or less word for word the same as the argument as in Proposition 2, Appendix, Chapter VII of loc. cit., and consists of a series of fairly straightforward checks.

Recall that if $U$ is a unipotent group scheme, we define $U^{n}$ inductively by $U^{1}=[U, U]$ and $U^{n}=\left[U^{n-1}, U\right]$ and $U_{n}$ by $U_{n}=U / U^{n}$. Since $U$ is unipotent over $K$, a field of characteristic zero we know that each $U^{n} / U^{n+1}$ is a vector scheme, and that $U=U_{N}$ for large enough $N$.

TheOREm 3.44. Let $U$ be a unipotent group scheme acted on by G. Assume that $G$ is affine, and for all $n \geq 1, H^{0}\left(G, U^{n} / U^{n+1}\right)=0$. Then for all $n \geq 0$ the functor $\underline{H}^{1}\left(G, U_{n}\right)$ is represented by an affine scheme over $K$. In particular, the functor $\underline{H}^{1}(G, U)$ is represented by an affine scheme over $K$.

Proof. Note that the hypotheses imply that $\underline{H}^{0}\left(G, U^{n} / U^{n+1}\right)(R)=0$ for all $K$ algebras $R$, and hence, by induction on $n$, that $\underline{H}^{0}\left(G, U_{n}\right)(R)=0$ for all $K$-algebras $R$ and all $n \geq 1$. 
We will prove the theorem by induction on $n$, and our argument is almost word for word that given by Kim in the proof of Proposition 2, Section 1 of [28]. When $n=1, U_{1}$ is just a vector scheme associated to a representation of $G$, and we already know that $\underline{H}^{i}\left(G, U_{1}\right)$ is representable for all $i$. For general $n \geq 1$, we know that we have an exact sequence

$$
1 \rightarrow U^{n+1} / U^{n+2} \rightarrow U_{n+1} \rightarrow U_{n} \rightarrow 1
$$

realising $U_{n+1}$ as a central extension of $U_{n}$ by the vector scheme $U^{n+1} / U^{n+2}$, and we may choose a splitting $s: U_{n} \rightarrow U_{n+1}$ of this exact sequence (just as a map of schemes). Looking at the long exact sequence in cohomology associated to this exact sequence, the boundary map $\underline{H}^{1}\left(G, U_{n}\right) \rightarrow \underline{H}^{2}\left(G, U^{n+1} / U^{n+2}\right)$ map between representables (using the induction hypothesis for representability of $\underline{H}^{1}\left(G, U_{n}\right)$ ) and hence the pre-image of $0 \in \underline{H}^{2}\left(G, U^{n+1} / U^{n+2}\right)$ is an (affine) closed sub-scheme of $\underline{H}^{1}\left(G, U_{n}\right)$, which we will denote by $I\left(G, U_{n}\right)$. Thus we get a vector scheme $\underline{H}^{1}\left(G, U^{n+1} / U^{n+2}\right)$, an affine scheme $I\left(G, U_{n}\right)$, and an exact sequence

$$
1 \rightarrow \underline{H}^{1}\left(G, U^{n+1} / U^{n+2}\right)(R) \rightarrow \underline{H}^{1}\left(G, U_{n+1}\right)(R) \rightarrow I\left(G, U_{n}\right)(R) \rightarrow 1
$$

for all $R$, in the sense of Proposition 3.43. We claim that in fact this sequence is exact in the stronger sense that $\underline{H}^{1}\left(G, U^{n+1} / U^{n+2}\right)(R)$ acts freely on $\underline{H}^{1}\left(G, U_{n+1}\right)(R)$ (functorially in $R$ ), and the surjection $\underline{H}^{1}\left(G, U_{n+1}\right)(R) \rightarrow I\left(G, U_{n}\right)(R)$ identifies $I\left(G, U_{n}\right)(R)$ with the set of orbits for this action. We will give the argument for $K$ points, since functoriality will be clear from the definition of the action, and the case of $R$-points is handled identically.

Since $U^{n+1} / U^{n+2}$ is in the centre of $U_{n+1}$, there is an action of the group $Z^{1}\left(G, U^{n+1} / U^{n+2}\right)$ of cocyles on $Z^{1}\left(G, U_{n+1}\right)$ by $(\sigma * \phi)(g)=\sigma(g) \phi(g)$ for $\phi \in$ $Z^{1}\left(G, U^{n+1} / U^{n+2}\right), \sigma \in Z^{1}\left(G, U_{n+1}\right)$ and $g \in G(T)$. It is straightforward to check that this descends to an action of $H^{1}\left(G, U^{n+1} / U^{n+2}\right)$ on $H^{1}\left(G, U_{n+1}\right)$. Suppose that $\sigma, \sigma^{\prime} \in Z^{1}\left(G, U_{n+1}\right)$ represent cohomology classes in the same orbit under this action. Thus there exists some $\phi \in Z^{1}\left(G, U^{n+1} / U^{n+2}\right)$ and $u \in U_{n+1}(L)$ such that

$$
\sigma(g) \phi(g)=\left(t(g)^{*} u\right)^{-1} \sigma^{\prime}(g) \rho(g)\left(s(g)^{*} u\right)
$$

for all $g \in G(T)$. If we now project to $U_{n}$ we can see that the cohomology classes of $\sigma$ and $\sigma^{\prime}$ map to the same element of $H^{1}\left(G, U_{n}\right)$. This argument can be reversed, and so the orbits for this action can be exactly identified with $I\left(G, U_{n}\right)(K)$.

To show freeness of the action, suppose that the cohomology class of $\phi \in Z^{1}\left(G, U^{n+1} / U^{n+2}\right)$ stabilises the cohomology class of $\sigma \in Z^{1}\left(G, U_{n+1}\right)$, so that

$$
\sigma(g) \phi(g)=\left(t(g)^{*} u\right)^{-1} \sigma(g) \rho(g)\left(s(g)^{*} u\right)
$$

for some $u \in U_{n+1}(L)$. Now, projecting to $U_{n}$ gives

$$
t(g)^{*} \bar{u}=\bar{\sigma}(g) \rho(g)\left(s(g)^{*} \bar{u}\right) \bar{\sigma}(g)^{-1}
$$

where we have written $\bar{u}($ resp. $\bar{\sigma})$ for the image of $u$ (resp. $\sigma)$ in $U_{n}\left(\operatorname{resp} . Z^{1}\left(G, U_{n}\right)\right.$ ). Now an easy induction using the fact that $H^{0}\left(G, U^{m} / U^{m+1}\right)=0$ for all $m \geq 1 \mathrm{im}$ - 
plies that $\bar{u}=1$, and thus, since $U^{n+1} / U^{n+2}$ is central in $U_{n+1}$, that $\sigma(g)=$ $\left(t(g)^{*} u\right)^{-1} \rho(g)\left(s(g)^{*} u\right)$ is a coboundary.

If we take $R$ to be the co-ordinate ring of $\underline{H}^{1}\left(G, U_{n}\right)$ and lift the identity in $\underline{H}^{1}\left(G, U_{n}\right)(R)$ via the surjective map

$$
\underline{Z}^{1}\left(G, U_{n}\right)(R) \rightarrow \underline{H}^{1}\left(G, U_{n}\right)(R)
$$

we get a splitting

$$
i: \underline{H}^{1}\left(G, U_{n}\right) \rightarrow \underline{Z}^{1}\left(G, U_{n}\right)
$$

and composing with the map $s: \underline{C}^{1}\left(G, U_{n}\right) \rightarrow \underline{C}^{1}\left(G, U_{n+1}\right)$ and the boundary map $d: \underline{C}^{1}\left(G, U_{n+1}\right) \rightarrow \underline{C}^{2}\left(G, U_{n+1}\right)$ gives a map

$$
d s i: \underline{H}^{1}\left(G, U_{n}\right) \rightarrow \underline{C}^{2}\left(G, U_{n+1}\right)
$$

which factors through $\underline{Z}^{2}\left(G, U^{n+1} / U^{n+2}\right)$, and is such that the induced map $\underline{H}^{1}\left(G, U_{n}\right) \rightarrow \underline{H}^{2}\left(G, U^{n+1} / U^{n+2}\right)$ is the boundary map appearing in the long exact sequence of cohomology from Proposition 3.43. Hence we have

$$
I\left(G, U_{n}\right)=(d s i)^{-1}\left(\underline{B}^{2}\left(G, U^{n+1} / U^{n+2}\right)\right) .
$$

Now, the proof of Proposition 3.41 shows that the functors $\underline{C}^{1}\left(G, U^{n+1} / U^{n+2}\right)$ and $\underline{B}^{2}\left(G, U^{n+1} / U^{n+2}\right)$ are represented by the vector schemes associated to the $K$ vector spaces

$$
\begin{aligned}
& \underline{C}^{1}\left(G, U^{n+1} / U^{n+2}\right)(K)=C^{1}\left(G, U^{n+1} / U^{n+2}\right) \\
& \underline{B}^{2}\left(G, U^{n+1} / U^{n+2}\right)(K)=B^{2}\left(G, U^{n+1} / U^{n+2}\right)
\end{aligned}
$$

respectively, and hence we may choose a functorial splitting

$$
a: \underline{B}^{2}\left(G, U^{n+1} / U^{n+2}\right) \rightarrow \underline{C}^{1}\left(G, U^{n+1} / U^{n+2}\right)
$$

and can define a map $b: I\left(G, U_{n}\right) \rightarrow \underline{C}^{1}\left(G, U_{n+1}\right)$ by $b(x)=(s i)(x)((a d s i)(x))^{-1}$. Then another explicit calculation shows that $b$ factors throughout $\underline{Z}^{1}\left(G, U_{n+1}\right)$, and the induced map $I\left(G, U_{n}\right) \rightarrow \underline{H}^{1}\left(G, U_{n+1}\right)$ is a splitting of the natural surjection $\underline{H}^{1}\left(G, U_{n+1}\right) \rightarrow I\left(G, U_{n}\right)$.

Thus using the stronger sense in which the sequence (148) is exact, we get an isomorphism of functors

$$
\underline{H}^{1}\left(G, U_{n+1}\right) \cong \underline{H}^{1}\left(G, U^{n+1} / U^{n+2}\right) \times I\left(G, U_{n}\right)
$$

and hence $\underline{H}^{1}\left(G, U_{n+1}\right)$ is represented by an affine scheme over $K$.

Corollary 3.45. With the assumptions as in the previous theorem, assume further that $H^{1}\left(G, U^{i} / U^{i+1}\right)$ is finite dimensional for each $n$. Then $\underline{H}^{1}\left(G, U_{n}\right)$ is of finite type over $K$, of dimension at most $\sum_{i=1}^{n-1} \operatorname{dim}_{K} H^{1}\left(G, U^{i} / U^{i+1}\right)$. 
Recall that for a 'good' morphism $f: X \rightarrow S$ over a finite field satisfying Hypothesis 3.4 , we have the period map

$$
X(S) \rightarrow H_{\text {rig }}^{1}\left(S, \pi_{1}(X / S, p)_{n}\right)
$$

taking a section to the corresponding path torsor. Choosing a closed point $s \in S$ means we can interpret this map as

$$
X(S) \rightarrow H^{1}\left(\underline{\operatorname{Aut}}_{K}^{\otimes}\left(s^{*}\right), \pi_{1}^{\mathrm{rig}}\left(X_{s}, p(s)\right)_{n}\right) .
$$

This latter set has the structure of an algebraic variety over $K$ under the condition that

$$
H_{\text {rig }}^{0}\left(S, \pi_{1}^{\text {rig }}(X / S, p)^{n} / \pi_{1}^{\text {rig }}(X / S, p)^{n+1}\right)
$$

is zero for each $n$. If, for example, $X$ is a model for a smooth projective curve $C$ over a function field, then we expect this condition to be satisfied under certain non-isotriviality assumptions on the Jacobian of $C$.

Acknowledgments. This paper is intended to form part of the author's PhD thesis, and was written under the supervision of Ambrus Pál at Imperial College, London, funded by an EPSRC Doctoral Training Grant. He would like to thank Dr. Pál both for suggesting the direction of research and for providing support, encouragement and many hours of fruitful discussions, without which this paper would not have been written.

He would also like to thank the anonymous referee for a careful reading of the paper, and in particular suggesting how to extend the main theorem from smooth affine curves to higher dimensions.

\section{REFERENCES}

[1] T. ABE, Explicit calculation of Frobenius isomorphisms and Poincaré duality in the theory of arithmetic $\mathscr{D}$-modules, preprint (2011), arXiv:math/1105.5796.

[2] P. BERTHELOT, Cohomologie rigide et cohomologie rigide à supports propres, première partie, preprint (1996).

[3] P. Berthelot, $\mathscr{D}$-modules arithmétique I. Opérateurs différentiels de niveau fini, Ann. scient. Éc. Norm. Sup. 29 (1996), 185-272.

[4] P. Berthelot, $\mathscr{D}$-modules arithmétique II. Descente par Frobenius, Mémoires de la SMF (2000), no. 81.

[5] P. Berthelot, Introduction à la théorie arithmétique des $\mathscr{D}$-modules, Cohomologies p-adiques et applications arithmétiques, II, no. 279, Astérisque, 2002, pp. 1-80.

[6] D. CARo, D-modules arithmétiques surcohérents. Applications aux fonctions L, Ann. Inst. Fourier, Grenoble 54 (2004), no. 6, 1943-1996.

[7] D. CARo, Dévissages des F-complexes de $\mathcal{D}$-modules arithmétiques en $F$-isocristaux surconvergents, Inventiones math. 166 (2006), 397-456.

[8] D. CARo, F-isocristaux surconvergents et surcohérence differentielle, Inventiones math. 170 (2007), 507-539.

[9] D. Caro, D-modules arithmétiques surholonomes, Ann. scient. Éc. Norm. Sup. 42 (2009), no. 1, 141-192.

[10] D. CARo, Stabilité par produit tensoriel de la surholonomie, preprint (2012), arXiv: math/0605.125v5. 
[11] D. CARo, Pleine fidélité sans structure de Frobenius et isocristaux partiellement surconvergents, Math. Ann. 349 (2011), no. 4, 747-805.

[12] D. CARo, Sur la préservation de la surconvergence par l'image directe d'un morphisms propre et lisse, preprint (2012), arXiv:math/0811.4740v3.

[13] D. CARo - N. TsuzuKI, Overholonomicity of overconvergent F-isocrystals over smooth varieties, Ann. of Math. 176 (2012), no. 2, 747-813.

[14] C. Chabauty, Sur les points rationnels des courbes algébriques de genre supérieur à l'unité, C. R. Acad. Sci. Paris 212 (1941), 882-885.

[15] B. ChiARELlotTo, Weights in rigid cohomology applications to unipotent F-isocrystals, Ann. scient. Éc. Norm. Sup. 31 (1998), 683-715.

[16] B. Chiarellotto and B. Le Stum, F-isocristaux unipotents, Compositio Math. 116 (1999), 81-110.

[17] B. Chiarellotto and B. Le Stum, Pentes en cohomologie rigide et F-isocristaux unipotents, Manuscripta Math. 100 (1999), 455-468.

[18] P. Deligne, Equations différentielles à points singuliers réguliers, Lecture Notes in Mathematics, vol. 168, Springer-Verlag, New York, 1970.

[19] P. Deligne, Le groupe fondamental de la droite projective moins trois points, Galois Groups over Q, Math. Sci. Res. Inst. Pub., 1989, pp. 79-297.

[20] P. Deligne, Catégories tannakiennes, The Grothendieck Festschrift, Vol. II, Progress in Mathematics, vol. 87, Birkhäuser, 1990.

[21] A. Dimca - F. MaAref - C. SABbah - M. SAito, Dwork cohomology and algebraic Dmodules, Math. Ann. 318 (2000), no. 1, 107-125.

[22] H. Esnault - P. HaI - X. Sun, On Nori's fundamental group scheme, Geometry and Dynamics of Groups and Spaces, Progress in Mathematics, vol. 265, Birkhäuser, 2007, pp. 377-398.

[23] A. Borel et al., Algebraic D-modules, Perspectives in Mathematics, vol. 2, Academic Press Inc., 1987.

[24] M. Hadian-JazI, Motivic fundamental groups and integral points, Ph.D. thesis, Universität Bonn, 2010.

[25] R. HAIN - S. ZUCKER, Unipotent variations of mixed Hodge structure, Inventiones math. 88 (1987), 83-124.

[26] R. Hartshorne, On the de Rham cohomology of algebraic varieties, Publ. Math. I.H.E.S. 45 (1975), no. 1, 6-99.

[27] N. KaTz, Nilpotent Connections and the Monodromy Theorem: Applications of a result of Turrittin, Publ. Math. I.H.E.S. 39 (1970), 175-232.

[28] M. Kim, The motivic fundamental group of $\mathbb{P}^{1} \backslash\{0,1, \infty\}$ and the theorem of Siegel, Inventiones math. 161 (2005), 629-656.

[29] M. Kim, The unipotent Albanese map and Selmer varieties for curves, Publ. RIMS, Kyoto Univ. 45 (2009), 89-133.

[30] S. MacLane, Categories for the working mathematician, Graduate Texts in Mathematics, vol. 5, Springer, 1971.

[31] J.S. Milne and P. Deligne, Tannakian categories, Hodge Cycles, Motives and Shimura Varieties, Lecture Notes in Mathematics, vol. 900, Springer, 1981, pp. 101-228.

[32] V. NAVARro Aznar, Sur la connection de Gauss-Manin en homotopie rationelle, Ann. scient. Éc. Norm. Sup. 26 (1993), 99-148.

[33] J.-P. Serre, Local Fields, Translated from the French by Marvin Jay Greenberg, Graduate Texts in Mathematics, vol. 67, Springer-Verlag, 1979.

[34] A. TAMagawa, Finiteness of isomorphism classes of curves in positive characterisitc with prescribed fundamental groups, J. Algebraic Geom. 13 (2004), 675-724.

[35] J. Wildeshaus, Realisations of polylogarithms, Lecture Notes in Mathematics, vol. 1650, Springer, 1997.

Manoscritto pervenuto in redazione il 26 agosto 2013. 
